\title{
The powers of monodromy
}

\author{
Liam McAllister, ${ }^{a}$ Eva Silverstein, ${ }^{b, c, d}$ Alexander Westphal ${ }^{e}$ and Timm Wrase ${ }^{b}$ \\ ${ }^{a}$ Department of Physics, Cornell University, \\ Ithaca, NY 14853, U.S.A. \\ ${ }^{b}$ Stanford Institute for Theoretical Physics, Stanford University, \\ Stanford, CA 94305, U.S.A. \\ ${ }^{c} S L A C$ National Accelerator Laboratory, \\ 2575 Sand Hill Rd., Menlo Park, CA 94025, U.S.A. \\ ${ }^{d}$ Kavli Institute for Particle Astrophysics and Cosmology, \\ Stanford, CA 94305, U.S.A. \\ ${ }^{e}$ Deutsches Elektronen-Synchrotron DESY, Theory Group, \\ D-22603 Hamburg, Germany \\ E-mail: McAllister@Cornell.edu, evas@slac.stanford.edu, \\ alexander.westphal@gmail.com, timm.wrase@stanford.edu
}

ABSTRACT: Flux couplings to string theory axions yield super-Planckian field ranges along which the axion potential energy grows. At the same time, other aspects of the physics remain essentially unchanged along these large displacements, respecting a discrete shift symmetry with a sub-Planckian period. After a general overview of this monodromy effect and its application to large-field inflation, we present new classes of specific models of monodromy inflation, with monomial potentials $\mu^{4-p} \phi^{p}$. A key simplification in these models is that the inflaton potential energy plays a leading role in moduli stabilization during inflation. The resulting inflaton-dependent shifts in the moduli fields lead to an effective flattening of the inflaton potential, i.e. a reduction of the exponent from a fiducial value $p_{0}$ to $p<p_{0}$. We focus on examples arising in compactifications of type IIB string theory on products of tori or Riemann surfaces, where the inflaton descends from the NS-NS two-form potential $B_{2}$, with monodromy induced by a coupling to the R-R field strength $F_{1}$. In this setting we exhibit models with $p=2 / 3,4 / 3,2$, and 3 , corresponding to predictions for the tensor-to-scalar ratio of $r \approx 0.04,0.09,0.13$, and 0.2 , respectively. Using mirror symmetry, we also motivate a second class of examples with the role of the axions played by the real parts of complex structure moduli, with fluxes inducing monodromy.

KEYwords: Cosmology of Theories beyond the SM, Flux compactifications

ARXIV EPRINT: 1405.3652 


\section{Contents}

1 Motivation and overview 1

2 Flux couplings and monodromy $\quad \mathbf{5}$

2.1 Axions from the two-form potential $B \quad 5$

$\begin{array}{ll}2.2 \text { Radiative stability } & 10\end{array}$

$\begin{array}{lll}2.3 & \text { Dual axions } & 10\end{array}$

3 Monodromies of Neveu-Schwarz $B$ fields $r$

3.1 Complex structure adjustment along a $B$ axion trajectory 11

$\begin{array}{lll}3.2 & \text { Kinetic energies } & 14\end{array}$

$\begin{array}{lll}3.3 & \text { Transverse axion directions } & 16\end{array}$

4 Embedding in Riemann surface compactifications $\quad 16$

$\begin{array}{llr}4.1 \text { A concrete setup } & 16\end{array}$

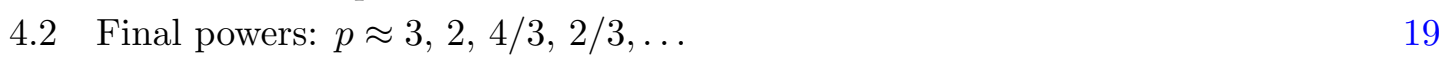

5 Monodromies of complex structure moduli 25

6 Conclusions 25

\section{Motivation and overview}

Cosmological observables provide a window into very early times in our universe, offering a unique set of probes of high-energy physics. In particular, in the context of inflation [2-5], an observation of a tensor-to-scalar ratio $r \gtrsim 10^{-2}$ implies an unprecedented connection between empirical observations and quantum gravity, for two reasons: it provides a measurement of the quantum mechanical variance of the tensor modes of the metric [6-13], and it indicates a super-Planckian field excursion [14, 15]. An impressive variety of observational efforts are approaching the sensitivity required to detect $r$ in this range [16], with a recent report of a detection of B-mode polarization $[17,18]$ that may contain a signal of primordial origin corresponding to inflationary tensor modes [19, 20], depending on the outcome of important foreground measurements generalizing [21, 22].

The inflationary energy density in large-field inflation is sub-Planckian - albeit relatively high, $\sim\left(10^{16} \mathrm{GeV}\right)^{4}$ - so that the process can be described, and was originally discovered theoretically, in the context of low-energy quantum field theory coupled to gravity. But the large field range implies sensitivity to an infinite sequence of dangerously irrelevant Planck-suppressed operators. Low-energy field theory models of large-field inflation can be radiatively stable, and natural in the sense of Wilsonian renormalization, by virtue of an approximate shift symmetry. However, imposing such a symmetry, even at the classical 
level, amounts to making a strong assumption about the ultraviolet (UV) completion of the inflationary effective theory. It would be much more satisfying - and in our view it is necessary - to understand how the structures required for large-field inflation emerge from a complete theory of quantum gravity.

It is tempting to belabor the motivation for modeling inflation in string theory by drawing examples from other subjects, such as condensed matter physics, illustrating the importance of the 'ultraviolet'-complete treatment in the presence of sensitivity to irrelevant operators in the effective theory. ${ }^{1}$ Even though one can model certain low-energy phenomena such as superconductivity using a continuum field theory, knowledge of the microphysics is required to understand very basic aspects of the problem, such as the transition temperatures available in real materials. For example, in applying BCS theory to metals one needs to recognize that the attractive interaction yielding Cooper pairs arises from phonons. Low-energy theory alone would suggest a much wider variety of transition temperatures than is observed in nature, a discrepancy that may be due to constraints from the UV completion of the system. More generally, important aspects of the physics (such as transport) can be described by an irrelevant operator, and thus be sensitive to aspects of the UV theory (such as the breakdown of translation invariance due to the lattice). Moreover, certain effects, such as the melting of a solid, are strongly UV sensitive. Although one can model a wide variety of behaviors in low-energy field theory, it would be a mistake to work purely in a low-energy effective description, ignoring the structure and constraints implied by the ultraviolet theory.

Of course, the major difference in the present case is that we do not know the correct theory of quantum gravity, whereas in the condensed matter analogue the relevant short distance theory is standard. But that is a logically independent point, and does not diminish the importance of obtaining large-field inflation from a complete theory of quantum gravity.

Without detailed knowledge of the UV completion of gravity, one might worry that as the inflaton moves over a Planckian range in field space - or more generally a range $M_{\mathrm{UV}} \leq M_{P}$, where $M_{\mathrm{UV}}$ characterizes the scale of new physics involved in quantum gravity - new degrees of freedom could become important in the dynamics. These new degrees of freedom could be different in different parts of the long field excursion, and lead to independent contributions to the potential that strongly violate the slow roll conditions. Note that this is already an important question at the classical level: although a shiftsymmetric model can be radiatively stable, and hence internally consistent from the lowenergy effective field theory point of view, whether a given shift symmetry admits an ultraviolet completion in quantum gravity requires careful examination.

String theory is a very promising candidate theory of quantum gravity, with many concrete successes in the arena of thought experiments and mathematical and physical consistency checks. The strong evidence for its consistency includes precise black hole entropy counts, the AdS/CFT correspondence, the perturbative finiteness of the theory, its role in resolving singularities, the intricate duality relations that make sense of various

\footnotetext{
${ }^{1}$ We thank S. Hartnoll and S. Kachru for discussions.
} 

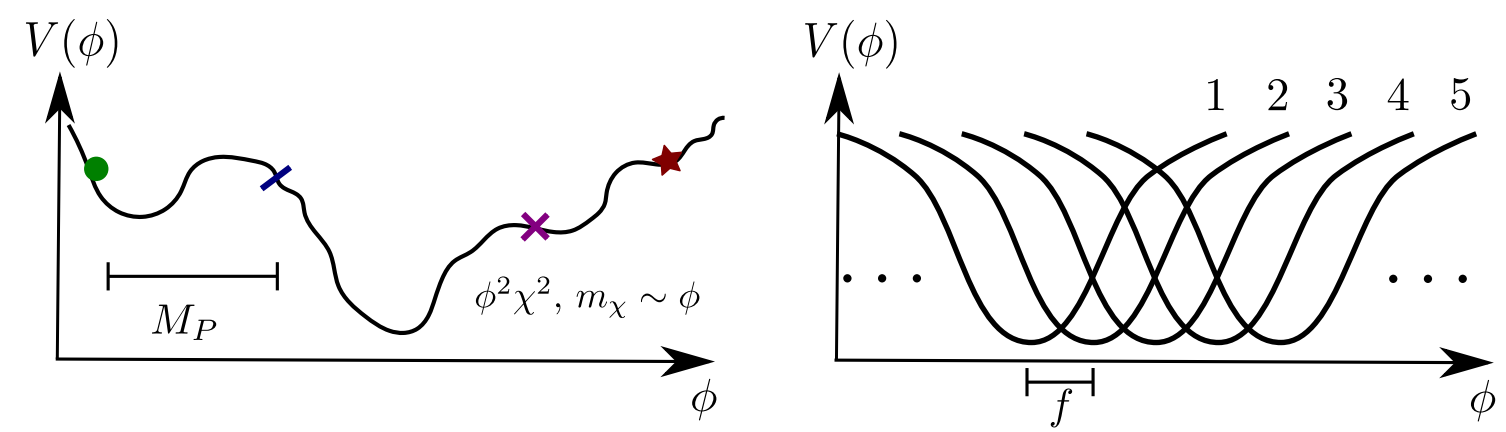

Figure 1. On the left, a sketch of a large field range with new effects - such as altered couplings or new light states - appearing after each displacement of order $\sim M_{P}$, parameterizing our ignorance of quantum gravity. Such features could arise both at the classical and the quantum level. On the right, the structure of the potential along axion directions (and their various duals) in string theory. The whole structure has a sub-Planckian period $f$, but on each branch the field can reach a large field range. The potential energy grows with each cycle around the underlying period $f$, while other conditions — such as the spectrum of branes wrapping the cycles threaded by the higher dimensional potential fields yielding axions — remain the same each time around. This suppresses many dangerous effects and leads to a controllable large field range. The result is a radiatively-stable potential as in chaotic inflation with a monomial potential.

strong coupling and high-curvature limits, and the capacity of its landscape of vacua to accommodate the small cosmological constant (as a selection effect). Despite the astronomical number of solutions of string theory, the mathematical structure of the theory remains highly constrained.

The microphysical structure of string theory provides a rather simple and general mechanism for large-field inflation [23], monodromy [24-29], in which an underlying periodicity of the theory ensures that as the inflaton field traverses many cycles with sub-Planckian period $2 \pi f \ll M_{P}$, the potential energy increases over each cycle but much of the remaining physics essentially repeats itself (see figure 1).

In this work, we will begin a more systematic analysis of the monodromy effect in string theory and its application to inflationary cosmology. As we will review in detail below, the couplings of axions to fluxes exhibit monodromy in a robust way. In the presence of sufficiently generic fluxes or brane configurations, the field ranges of axion fields (and their duals) extend to super-Planckian values, but the underlying sub-Planckian periodicity governs much of the physics along the trajectory [24-29] including significant sectors of the spectrum of particles and branes. Starting from this general framework, we will provide a new class of specific examples of monodromy inflation in string theory, with a variety of values of the tensor-to-scalar ratio, including some with significantly larger values of $r$ than in previously studied realizations of monodromy in string theory. The cause of the large values of $r$ is very simple: in our examples, inflation is driven by potential energy terms that involve moderately high powers of the axion field.

Although the monodromy phenomenon in itself is quite simple, there are substantial complications involved in modeling inflation explicitly in string theory: a primary problem is the stabilization of the many moduli fields that arise upon compactification. In the 
new models presented here, moduli stabilization is simpler in one respect than in previous realizations: the inflationary energy itself plays a leading role in determining the vevs of some of the moduli, which shift adiabatically as inflation proceeds. This flexibility allows for successful moduli stabilization even when the inflationary energy is large enough to invalidate a more rigid stabilization scenario. Moreover, the shifting of the moduli alters the form of the inflaton potential, while not disrupting inflation. With the adjustment of the moduli fields consistently taken into account, we find potentials that take the form of a sinusoidally modulated power law,

$$
V(\phi) \approx \mu^{4-p} \phi^{p}+\Lambda^{4} \sin \left(\frac{\phi}{f}\right),
$$

over the relevant range of the inflaton field $\phi$. Previous work had exhibited a concrete example with $p=1[25,26]$, and motivated a variety of others with various powers $p[25-$ 47]. In the present work, we will derive a broader range of powers $p$ from a wider variety of flux-induced axion interactions. ${ }^{2}$

The fields of primary interest here arise from the internal components of higher dimensional potential fields (of various ranks), which are generalizations of the vector potential $A$ of electromagnetism. The axions descend from rank- $p$ potential fields $A_{p}$ as $A_{p}=a \omega_{p}$, where $\omega_{p}$ is a $p$-form in the cohomology of the internal space. For example, a one-form $A$ integrated around a circle $S^{1}$ in the extra dimensions gives an axion, $a=\int_{S^{1}} A$. There is a rich set of gauge invariant terms in the low-energy Lagrangian of compactified string theory that exhibit direct dependence on these potential fields and on their field strengths; these generalize Stueckelberg terms $(\partial C+A)^{2}$ that are gauge-invariant under $A \rightarrow A+\partial \Lambda, C \rightarrow C-\Lambda$.

In section 2, we will give an overview of the monodromy arising from the couplings between fluxes and axion fields in string theory, paying particular attention to the contributions relevant for our new examples. The couplings to fluxes of an axion field $a$ produce a potential that at large $a$ takes the form $V \sim f\left(\chi_{I}\right) \times a^{p_{0}}$ where the $\chi_{I}$ are moduli fields, as well as massive Kaluza-Klein scalars, and $p_{0}$ is an integer. Backreaction of the inflationary energy on the scalars $\chi_{I}$, which adjust in an energetically favorable way, can change the shape of the potential at large field values. Previous work focused on examples with $p_{0}=2$; in one canonical class of examples this fiducial power is 'flattened' to the linear potential $V \propto a^{1}$ originally studied in [25, 26], as can be seen explicitly using the gravity-side description of the corresponding brane system [30]. In the present work, we will include examples in which higher-rank wedge products of the rank-two potential field $B$ sourced by fundamental strings lead to higher fiducial powers $p_{0}$. In particular, we find examples with $p_{0}=4$. We will also comment on dual cases, including axion-like components of complex structure moduli of Calabi-Yau and Riemann surface compactifications. After an instructive warmup example in section 3 exhibiting flattening along a complex structure direction, we will present string compactifications that realize monodromy inflation in section 4 . In these examples, the flattening effect leads to a variety of final powers

\footnotetext{
${ }^{2}$ Other interesting recent work on axion inflation in string theory without incorporating monodromy appears in [48-59], building on earlier works such as [60-64].
} 
including $p=3,2,4 / 3$ and $2 / 3$. In section 5 , we will make comments on the monodromies in complex structure moduli space from flux potentials in Calabi-Yau and Riemann surface compactifications.

\section{Flux couplings and monodromy}

In this section we explain the origin of terms in the effective action that have a monodromyinduced potential growing as an integer power $p_{0} \geq 2$ of an axion field, while also depending on moduli fields coming from the internal metric and the dynamical string coupling. In section 3, we will show explicitly how these terms can lead to a variety of power law potentials $V \propto \phi^{p \leq p_{0}}$, with the final power $p$ shifted down from $p_{0}$ via adjustments of heavy moduli.

\subsection{Axions from the two-form potential $B$}

Perturbative string theory contains a two-form potential field $B=B_{M N} d x^{M} \wedge d x^{N}$ that is directly analogous to the usual vector potential $A=A_{M} d x^{M}$ of electromagnetism. ${ }^{3}$ In particular, $B$ is sourced by fundamental strings just as the usual vector potential is sourced by charged particles. There is a gauge invariance in the theory under which $B \rightarrow B+d \Lambda_{1}$, with $\Lambda_{1}$ a one-form, analogous to the gauge invariance under $A \rightarrow A+d \Lambda_{0}$ in electromagnetism. Similarly, there are other potential fields denoted $C_{p+1}$ sourced by $p$-dimensional extended objects (D $p$-branes) [65].

In electromagnetism, the action contains the gauge-invariant terms

$$
S_{\mathrm{EM}}=\int d^{4} x \sqrt{-g}\left\{F_{M N} F^{M N}-\rho^{2}\left(A_{M}+\partial_{M} C\right)^{2}+\ldots\right\},
$$

where under the gauge transformation $A_{M} \rightarrow A+\partial_{M} \Lambda_{0}$, the field $C$ transforms as $C \rightarrow$ $C-\Lambda_{0}$. The first term is the Maxwell action, written in terms of the field strength $F=d A$. The second term, known as a Stueckelberg term, can arise from spontaneous symmetry breaking, with $\rho$ the vacuum expectation value of a charged field. ${ }^{4}$

In type II string theory, one finds generalizations of these Maxwell and Stueckelberg terms, with the gauge transformation $B \rightarrow B+d \Lambda_{1}$ accompanied by appropriate shifts of the $C_{p}$ fields. Although we will focus on specific examples in type IIB string theory below, let us start by considering the relevant terms arising in $D=10$ type IIA string theory. There we have potential fields $C_{p}$ with odd $p$, and it is useful to define the following generalized field strengths that respect all the gauge symmetries of the theory:

$$
\begin{aligned}
H_{3} & =d B, \\
F_{0} & =Q_{0}, \\
\tilde{F}_{2} & =d C_{1}+F_{0} B, \\
\tilde{F}_{4} & =d C_{3}+C_{1} \wedge H_{3}+\frac{1}{2} F_{0} B \wedge B,
\end{aligned}
$$

\footnotetext{
${ }^{3}$ An exception is the type I string, in which closed strings are unstable to breaking into open strings, but this theory contains a two-form potential sourced by D1-branes.

${ }^{4}$ In ordinary electrodynamics the symmetry is of course unbroken in vacuum, but $\rho \neq 0$ arises in a superconductor from the condensation of the Cooper pair field.
} 
where $Q_{0}$ is an integer. These are gauge-invariant, with the transformation $B \rightarrow B+d \Lambda_{1}$ extended to a combined transformation

$$
\begin{aligned}
\delta B & =d \Lambda_{1}, \\
\delta C_{1} & =-F_{0} \Lambda_{1}, \\
\delta C_{3} & =-F_{0} \Lambda_{1} \wedge B .
\end{aligned}
$$

The effective action starting from a total dimensionality $D=10$ contains terms proportional to ${ }^{5}$

$$
-\frac{1}{\alpha^{\prime}} \int d^{10} x \sqrt{-G}\left\{\frac{1}{g_{s}^{2}}\left|H_{3}\right|^{2}+\sum_{p}\left|\tilde{F}_{p}\right|^{2}\right\} .
$$

Upon dimensional reduction to four dimensions, these terms introduce a direct dependence of the potential energy on the axion fields

$$
b^{i} \equiv \int_{\Sigma_{2}^{i}} B
$$

obtained by integrating the potential field $B$ over nontrivial 2-cycles $\Sigma_{2}^{i}$ in the compactification manifold $\mathcal{M}$. Another feature we need to take into account is that the fluxes $Q_{2}^{i}=\int_{\Sigma_{2}^{i}} d C_{1}, Q_{4}=\int_{\Sigma_{4}^{i}} d C_{3}$, and $N_{3}=\int_{\Sigma_{3}^{a}} H_{3}$ (with the index $i$ running over topologically distinct even-dimensional cycles, and $a$ similarly indexing three-cycles) are quantized, as is $Q_{0}=F_{0}$.

Let us focus on the $B$-dependent terms, and for simplicity work on the branch of the potential where $Q_{2}=Q_{4}=0$ (also setting to zero the flux $d C_{3}$ along the noncompact four dimensional spacetime, or equivalently the dual 6-form flux $Q_{6} \equiv \int_{\mathcal{M}} \star_{10} F_{4}=\int_{\mathcal{M}} F_{6}$ ). In the models in section 3 , we will incorporate the analogue in type IIB string theory of these additional fluxes, which will yield interesting behavior in some cases, but for now we will focus on the leading contributions to the potential at large field range. Given this, we have an action of the schematic form ${ }^{6}$

$$
-\frac{1}{\alpha^{\prime 4}} \int d^{10} x \sqrt{-G}\left\{\frac{1}{g_{s}^{2}}\left|H_{3}\right|^{2}+\left|Q_{0} B\right|^{2}+\left|Q_{0} B \wedge B\right|^{2}+\gamma_{4} g_{s}^{2}\left|Q_{0} B \wedge B\right|^{4}+\ldots\right\} .
$$

Here in the last term and the ellipses we have allowed for corrections that could be read off from the tree-level four-point and higher-point functions ( $\gamma_{4}$ being an order 1 number). We have also set to zero the contribution from $\left|\tilde{F}_{6}\right|^{2}=\left|C_{3} \wedge H_{3}+Q_{0} B \wedge B \wedge B / 6\right|^{2}$, having in mind situations where $H_{3}$ flux is present in order to contribute to moduli stabilization, and $C_{3}$ minimizes the $\left|\tilde{F}_{6}\right|^{2}$ term at zero. More generally, there should be interesting configurations in which $C_{3} \wedge H_{3} \neq-Q_{0} B \wedge B \wedge B / 6$ at the $C_{3}$ minimum, or configurations in which $C_{3}$ and $B$ evolve together, in which cases this term is relevant.

The field strengths of R-R terms come with a factor of $g_{s}$, so higher-dimension operators involving higher powers of generalized field strengths $\tilde{F}_{p}$ - even those from string

\footnotetext{
${ }^{5}$ Similar comments apply in the more generic cases with $D>10[66]$.

${ }^{6}$ See e.g. equation (12.1.25) of [65]. However, we caution the reader that we follow the sign conventions of [67], not those of [65].
} 
tree diagrams - appear with a relative factor of $Q_{0}^{2} g_{s}^{2}$, and are thus suppressed at small string coupling. This is in the standard frame we will use exclusively here, where gauge transformation and flux quantization conditions are most simply expressed.

In fact, there is generically an additional suppression factor at large radius. We will shortly consider generalizations that arise upon dimensional reduction or T-duality, where $F_{0}$ is replaced by higher-form fluxes $F_{n}$. In those cases, the suppression is even stronger, with each power of $|\tilde{F}|^{2}$ coming with a factor of $g_{s}^{2} Q_{n}^{2} / L^{2 n}$, where $L$ is the size in string units of the cycle threaded by the $F_{n}$ flux.

Below, we will consider specific examples in type IIB string theory with effective $\left|F_{1} B\right|^{2}+\left|F_{1} \wedge B \wedge B\right|^{2}$ interactions. These follow from T-duality of (2.6) upon reduction of the IIA theory on a circle as explained in detail in [67]. At first glance, this is not manifest from the generalized fluxes that appear in the type IIB equations of motion in ten dimensions:

$$
\begin{aligned}
H_{3} & =d B, \\
F_{1} & =d C_{0}, \\
\tilde{F}_{3} & =d C_{2}-C_{0} H_{3}, \\
\tilde{F}_{5} & =d C_{4}-\frac{1}{2} C_{2} \wedge H_{3}+\frac{1}{2} B \wedge d C_{2} .
\end{aligned}
$$

In $\tilde{F}_{5}$ we do not find an $F_{1} \wedge B \wedge B$ term by working directly in the ten-dimensional theory. However, T-duality on a circle, including the duality between D7-branes and D8-branes, requires this coupling to be present upon dimensional reduction. This indeed works out precisely [67]. Specifically, consider reducing ten-dimensional type IIB theory on a circle (along the $x^{9}$ direction, $x^{9} \cong x^{9}+2 \pi$ ), with

$$
\begin{aligned}
& C_{0}=x^{9} Q_{0}+\mathcal{C}_{0}, \\
& C_{2}=x^{9} Q_{0} B+\mathcal{C}_{2},
\end{aligned}
$$

where $\mathcal{C}_{p}$ are fluctuations of the potential fields about the background. Substituting (2.8) into (2.7), we find an effective $F_{1} \wedge B \wedge B$ contribution to $\tilde{F}_{5}$, and an effective $F_{1} \wedge B$ term in $\tilde{F}_{3}$. In the four-dimensional effective theory, there are many contributions of this kind, leading to axion potentials of the schematic form

$$
f\left(\chi_{I}\right) \frac{\left(Q^{(n)} a^{n}+Q^{(n-1)} a^{n-1}+\cdots+Q^{(0)}\right)^{2}}{L^{2 n^{\prime}}}+\cdots \sim \tilde{f}\left(\chi_{I}\right) a^{p_{0}} \quad \text { for } \quad a \gg 1,
$$

where we have denoted the axion field by $a, n=p_{0} / 2$ is a positive integer, and $\chi_{I}$ are the moduli fields, as well as additional scalar fields, whose important effects we will analyze below. The value of $n$ depends on the ranks of the fluxes and potential fields that descend to the $Q^{(i)}$ and to $a$, respectively; we will discuss specific examples in the following section.

We see immediately from (2.9) the branch structure of the monodromy-unwound potential: for a fixed value of the flux quantum number $Q^{(0)}$ here, the potential is a growing function of $a$, which has an unbounded field range (up to the point where the potential energy density becomes so large that the low-energy description breaks down). The whole 
structure, on the other hand, is periodic under shifts of $a$ by an integer, as this can be absorbed by an appropriate shift of the flux quantum numbers. That is, there is an identical branch of the potential for each value of $Q^{(0)}$, as in figure 1. Similarly, the spectrum of particles and higher dimensional branes that couple to $a$ is periodic ${ }^{7}$ under $a \rightarrow a+1$. For example, one gets D-strings from wrapping a D3-brane on a two-cycle threaded by $B$. A given wrapped D-brane gives a string in four dimensions with a tension that grows with the axion $b=\int B$, but the set of wrapped D-branes is invariant under shifts of $b$ by an integer. This provides a reasonably clean answer to the question of controlled large field ranges in quantum gravity.

As we will analyze in detailed examples below, the moduli-dependence in the potential energy has important effects on the inflationary dynamics. If the moduli (and other massive degrees of freedom, such as Kaluza-Klein modes, included in the $\chi_{I}$ ) are stabilized very rigidly, the inflationary potential could end up behaving like $a^{p_{0}}$. That requires the inflationary potential to be subdominant to the leading terms stabilizing the moduli. More generally, as we increase the vev of the axion field $a$, the other fields will adjust in response to the potential energy carried by the term (2.9). This can be an important effect even for fields more massive than the Hubble scale during inflation, as was first pointed out, and explored in various cases with $p_{0}=2$, in [30]. As an example, the linear potential of $[25,26]$ arises in a simple way as a flattening effect from $p_{0}=2$ to $p=1$.

The couplings we have reviewed above can produce examples in which the fiducial power $p_{0}$ is either 2 (e.g. from $\left|d C_{2} \wedge B\right|^{2}$ ) or 4 (e.g. from $\left|F_{n} \wedge B \wedge B\right|^{2}, n=0$ or 1 ). As we have seen, some of these couplings are manifest from a simple dimensional reduction of the terms in the higher-dimensional type II string theory action [65]. Other such terms come from appropriate field configurations, as in (2.8), that can arise in the reduction of the higher-dimensional theory. Some of these two types of terms, and many others, can be related to each other by duality symmetries.

A rich set of string dualities also relate the $B$ potential fields we have focused on here to the R-R potentials $C_{p}$, and also to other scalar fields such as the real parts of complex structure moduli (related to $B$ via T-duality or its generalizations like mirror symmetry). We will comment further on the latter case below. It would take us too far afield to enumerate all the possible fields and terms, but it is clear that the monodromy effect is ubiquitous - to avoid it requires turning off fluxes and/or choosing internal manifolds with special topology.

One final comment on genericity is worth making here: in this work we will consider string theory in $D=10$ dimensions, but $D>10$ limits of string theory also exhibit axionflux couplings with a similar structure, including important couplings to other scalar fields. In such cases, one might find even higher fiducial powers $p_{0}$, which when combined with

\footnotetext{
${ }^{7}$ The subsectors of the spectrum coming from wrapped branes as described in the text are periodic, while other sectors of metastable states can be affected by the monodromy along the branch of the potential on which the system inflates. See $[25,26]$ for explicit examples of both classes, with the latter case arising from modes living on a spacetime-filling brane. These latter sectors are the closest the system comes to the emergence of light states at large field [68], albeit not via an approach to a weak-coupling or large-radius limit of moduli space.
} 
heavy-field adjustments could lead to a larger range of potentials, which could be analyzed explicitly as in [66]. In fact, in $D>10$ the spectrum is dominated by axions from R$\mathrm{R}$ potential fields, whose number grows as $2^{D}$. In $D=10$, axions and their duals are an order-one fraction of the scalar fields, and hence already rather generic as candidate inflatons.

Before moving to specific examples in the next section, let us continue to study the general structure of the potential and how it behaves at large field range, in the regime where the canonically normalized field takes super-Planckian values. As already discussed, the axions $b_{i}$ from the two-form potential $B$ arise from cohomology elements in the internal manifold,

$$
B=\sum b_{i} \omega_{2}^{i},
$$

where $\omega_{2}^{i}$ are nontrivial two-forms. The relation between the axions $b_{i}$ and the canonical field depends on the geometry, and specifically on $\int \omega \wedge \star \omega$. For simplicity let us first consider a situation in which all length scales are comparable, of order $L$ in string units, and there is no significant warping. From the kinetic term $\int\left|H_{3}\right|^{2}$ for $B$ we get the canonical fields

$$
\phi_{i} \sim f b_{i} \sim b_{i} \frac{M_{P}}{L^{2}},
$$

and an effective action of the form

$$
\int d^{4} x \sqrt{-g}\left\{\sum_{i} g^{00} \dot{\phi}_{i}^{2}-V\left(\phi_{i} ; \chi_{I}\right)\right\}
$$

where $\chi_{I}$ denotes moduli fields and other degrees of freedom such as those related to the internal spatial profiles of the fields (i.e., Kaluza-Klein modes). With multiple fields there is the possibility of kinetic mixing, as we will discuss below.

With the above approximations we arrive at a potential of the form

$$
\begin{aligned}
V & \sim M_{P}^{4} \frac{g_{s}^{4}}{L^{6}} \frac{Q_{n}^{2}}{L^{2 n}}\left(\frac{b^{2}}{L^{4}}+\frac{b^{4}}{L^{8}}+\mathcal{O}\left(\frac{g_{s}^{2} Q_{n}^{2}}{L^{2 n}} \frac{b^{8}}{L^{16}}\right)\right) \\
& \sim M_{P}^{4} \frac{g_{s}^{4}}{L^{6}} \frac{Q_{n}^{2}}{L^{2 n}}\left(\frac{\phi^{2}}{M_{P}^{2}}+\frac{\phi^{4}}{M_{P}^{4}}+\mathcal{O}\left(\frac{g_{s}^{2} Q_{n}^{2}}{L^{2 n}} \frac{\phi^{8}}{M_{P}^{8}}\right)\right) .
\end{aligned}
$$

Here we have assumed that the configuration of fluxes and axion(s) $b_{i} \sim b$ is sufficiently generic so that $F_{n} \wedge B \wedge B \neq 0$, leading to a potential term quartic in $b$. In other special cases, e.g. in type IIB theory without the background field configuration in (2.8), the quartic term may be absent, leading to a quadratic fiducial potential.

The expression (2.13) has two main implications for our purposes. First, at least in this one-scale situation, the quartic term dominates in the super-Planckian regime $\phi \gg M_{P} .^{8}$ Second, the higher-dimension operators coming from higher powers of $\left|\tilde{F}_{p}\right|^{2}$ are negligible as long as $g_{s}^{2} / L^{2 n} \ll 1$. In $(2.13)$ we took into account that the largest power of $\phi / M_{P}$

\footnotetext{
${ }^{8}$ Although we have illustrated this point in a system with a single length scale, the result is more general. In fact, in configurations with multiple length scales, as described around (3.29) of [30], the higher powers of $\phi$ can dominate even for $\phi<M_{P}$.
} 
dominates in these terms suppressed by $g_{s}^{2} / L^{2 n}$. This requires moderately large radius and small string coupling. From now on, we will drop higher-dimension terms for this reason.

In the next section, we will incorporate backreaction on the moduli fields $\chi_{I}$, finding specific examples in which the fiducial power $p_{0}$ is shifted to various powers $p \leq p_{0}$ depending on the interplay of the leading large-field inflationary potential term and the other terms in the moduli potential,

$$
V \sim f\left(\chi_{I}\right) \phi^{p_{0}}+V_{0}\left(\chi_{I}\right) \rightarrow V(\phi) \approx \mu^{4-p} \phi^{p}+\Lambda^{4} \sin \left(\frac{\phi}{f}\right) .
$$

In the last term we allowed for periodic contributions, which are suppressed at large radius.

\subsection{Radiative stability}

For completeness, let us briefly review radiative stability in large-field inflation. Chaotic inflation with a monomial potential [23], including generalizations to non-integer powers $p$ via monodromy, as in (2.14), is radiatively stable. The couplings intrinsic to the power law potential (expanding in field perturbations $\delta \phi$ ) become smaller at large field range, and gravitational interactions are also suppressed [69]. In effect, as long as the inflationary scalar potential constitutes the leading source of shift symmetry breaking, the model is technically natural in the sense of 't Hooft (and can be fully natural in the sense of Wilson given dynamically small scales). On the other hand, establishing that the approximate shift symmetry encoded in (2.14) can arise in a consistent quantum gravity theory requires careful consideration of the ultraviolet completion: in the context of string compactifications, it is necessary in particular to verify that the symmetry survives stabilization of all moduli. We check this in explicit examples in section 4 below.

\subsection{Dual axions}

Before moving on to our main examples, we briefly mention other fields, related to the $B$ field by dualities, that undergo monodromy in the presence of appropriate fluxes. ${ }^{9}$

First, as noted above, string dualities relate the NS-NS two-form $B$ to the R-R $p$-forms $C_{p}$. We will not analyze such examples here, but characterizing them would be a large part of a systematic analysis of the monodromy mechanism. As an example, in one of the original models $[25,26]$ of axion monodromy, the inflaton is an axion descending from $C_{2}$.

Next, complex structure moduli of certain special compactification manifolds, such as Calabi-Yau manifolds and Riemann surfaces, contain components that behave like axions, i.e. fields that are periodic in the absence of monodromy-inducing sources. These are sometimes related by string dualities to the axions descending from higher dimensional components of the various gauge potentials. The motion of 7-branes is on the same footing in some sense, as 7-brane position moduli arise from complex structure moduli in F-theory. ${ }^{10}$

\footnotetext{
${ }^{9}$ Our discussion here is not exhaustive: other examples include configurations with moving branes [24, $32,33,35]$, as well as some of the scenarios in [36-47]; some of these may also be understood via dualities.

${ }^{10}$ See [70] for a recent paper that determines monodromies on $\mathrm{CY}_{4}$ manifolds.
} 
For the simplest example, consider string theory on a two-dimensional torus $T^{2}$. The complex structure modulus $\tau$ describes the same geometry if we shift $\tau \rightarrow \tau+1$. This complex structure modulus $\tau$ is related by a T-duality (or mirror symmetry) transformation to a modulus $\rho=b+i \operatorname{Vol}\left(T^{2}\right)$, where $b=\int_{T^{2}} B$ and $\operatorname{Vol}\left(T^{2}\right)$ is the volume of the twotorus. The underlying periodicity of $b$ is mirror to the transformation $\tau \rightarrow \tau+1$ of the real part of the complex modulus $\tau$. Flux threading one cycle of this $T^{2}$ removes the periodicity under $\tau \rightarrow \tau+1$, inducing monodromy. For Riemann surfaces with genus $h>1$, a similar effect arises, at least in a limit of complex structure where the surface nearly factorizes into $h$ tori separated by thin necks.

In Calabi-Yau manifolds, mirror symmetry relates the axions from $B$ to components of the complex structure moduli. The $B$ fields have an underlying periodicity, realized as a set of $\theta$ angles in a gauged linear sigma model [71] treatment of Calabi-Yau manifolds. The complex structure moduli exhibit a corresponding monodromy: the periods, and hence the fluxes, do not return to themselves after going around special points in the moduli space. About large complex structure, for example, there is a monodromy group $\mathbb{Z}$ as in the $T^{2}$ case just discussed.

The flux stabilization potential for complex structure moduli of Calabi-Yau manifolds [72] as well as of Riemann surfaces [73] contains a sextic potential for these complexstructure dual-axions, at fixed values of the remaining moduli. In some cases, such as the examples in [73], this flux potential for the complex structure moduli also provides a leading contribution to the stabilizing potential for the volume and the string coupling.

In section 5 we will remark briefly on the complex structure analogue (roughly the mirror) of the examples based on $B$ axions that appear in section 3 and section 4 . It would be worthwhile to analyze more systematically the possibility of complex structure monodromies for inflation.

\section{Monodromies of Neveu-Schwarz $B$ fields}

In this section we will illustrate the general considerations of section 2.1 in a concrete framework for moduli stabilization. We will exhibit a simple flattening effect [30] in which the axion potential energy participates in the stabilization of a complex modulus $u$, whose adjustment reduces the power in the axion potential from a fiducial value $p_{0}=4$ to $p=3$, at fixed values of the other moduli.

Later, in section 4, we will recover this effect within a class of string compactifications which also stabilize the volume and string coupling. In short, the inflationary axion will arise from the NS-NS $B$ field in compactification of type IIB string theory on a product of Riemann surfaces, $\Sigma_{1} \times \Sigma_{2} \times \Sigma_{3}$, with moduli stabilized as in [73] by a combination of fluxes and $(p, q) 7$-branes.

\subsection{Complex structure adjustment along a $B$ axion trajectory}

Consider type IIB string theory, but including the effective flux coupling T-dual to the term $\left|F_{0} B \wedge B\right|^{2}$ in the action (2.6) for massive type IIA string theory. As explained in the 
previous section, in the presence of background fields (2.8) [67], we effectively have a term in the type IIB action of the form

$$
S_{\text {IIB }} \supset \frac{1}{\alpha^{\prime 4}} \int\left|F_{1} \wedge B \wedge B\right|^{2}
$$

from the $\left|\tilde{F}_{5}\right|^{2}$ term. As usual in type IIB string theory, we must separately impose selfduality of $\tilde{F}_{5}[65] .^{11}$

We begin by studying compactification on the product of three two-tori, $\left(T^{2}\right)^{3}$, and later generalize to higher-genus Riemann surfaces. For simplicity we will take the tori to be rectangular, with metric

$$
d s^{2}=G_{m n} d y^{m} d y^{n}=\sum_{i=1}^{3} L_{1}^{2}\left(d y_{1}^{(i)}\right)^{2}+L_{2}^{2}\left(d y_{2}^{(i)}\right)^{2},
$$

with $y_{1} \equiv y_{1}+\sqrt{\alpha^{\prime}}, y_{2} \equiv y_{2}+\sqrt{\alpha^{\prime}}$, where $L_{1}$ and $L_{2}$ are dimensionless. Denote $L^{2}=L_{1} L_{2}$, so the total internal volume $\mathcal{V}$ is $L^{6} \alpha^{\prime 3}$. Introduce 3 -form flux

$$
F_{3}=(2 \pi)^{2} \frac{Q_{31}}{\sqrt{\alpha^{\prime}}} d y_{1}^{(1)} \wedge d y_{1}^{(2)} \wedge d y_{1}^{(3)}+(2 \pi)^{2} \frac{Q_{32}}{\sqrt{\alpha^{\prime}}} d y_{2}^{(1)} \wedge d y_{2}^{(2)} \wedge d y_{2}^{(3)},
$$

where the superscript labels the three two-tori and with $Q_{31}, Q_{32} \in \mathbb{Z}$. That is, we have $Q_{31}$ units of flux on the product of the three $y_{1}^{(i)}$ cycles and $Q_{32}$ units of flux on the product of the three $y_{2}^{(i)}$ cycles.

We now include quantized 1-form flux in the symmetric configuration

$$
F_{1}=\frac{Q_{1}}{\sqrt{\alpha^{\prime}}} \sum_{i=1}^{3} d y_{1}^{(i)}
$$

with $Q_{1} \in \mathbb{Z}$, so that $Q_{1}=\int d y_{1}^{(i)} F_{1}$.

The periods of $B$ on each individual $T^{2}$ give rise to the axions of primary interest:

$$
b^{(i)} \equiv \frac{1}{\alpha^{\prime}} \int_{T_{(i)}^{2}} B
$$

so that ${ }^{12}$

$$
B=\sum_{i=1}^{3} b^{(i)} d y_{1}^{(i)} \wedge d y_{2}^{(i)}+\ldots
$$

where the ellipses indicate additional axions from periods of $B$ on two-cycles consisting of pairs of one-cycles from two distinct tori. ${ }^{13}$

\footnotetext{
${ }^{11}$ The field configuration (2.8) also contributes to the Chern-Simons term $\int C_{4} \wedge H_{3} \wedge F_{3}$ in the type IIB action, but in the flux and axion backgrounds considered below, the relevant contribution $\int H_{3} \wedge F_{1} \wedge B$ will vanish.

${ }^{12}$ In our conventions, $b^{(i)}$ and $L$ are dimensionless, while $y_{i}$ have dimensions of length, and $B$ has the dimensions of length squared (so its components $B_{M N}$ are dimensionless, as are the components of the R-R potentials $C_{M_{1} \ldots M_{p}}^{(p)}$ ).

${ }^{13}$ Axions involving distinct tori could be projected out by a suitable orbifold action.
} 
We will first study the dynamics of the symmetric configuration

$$
b^{(1)}=b^{(2)}=b^{(3)} \equiv b,
$$

and will address the stability of the relative coordinates $b^{(i)}-b^{(j)}, i \neq j$, in section 3.3 below. Upon dimensional reduction, the four-dimensional Lagrange density for the scalars $b, L=\sqrt{L_{1} L_{2}}$ and $u=L_{2} / L_{1}$ takes the form (before converting to Einstein frame)

$$
\begin{aligned}
\mathcal{L}=\frac{a(t)^{3}}{\alpha^{\prime}}\{ & \frac{L^{6}}{g_{s}^{2}}\left(\frac{\dot{u}}{u}\right)^{2}+\frac{L^{6}}{g_{s}^{2}}\left(\frac{\dot{L}}{L}\right)^{2}+\frac{L^{6}}{g_{s}^{2}} \frac{\dot{b}^{2}}{L^{4}} \\
& \left.-\frac{L^{6}}{\alpha^{\prime}} \frac{Q_{1}^{2} u}{L^{2}}\left[\frac{b^{4}}{L^{8}}+\frac{b^{2}}{L^{4}}+1\right]-\frac{L^{6}}{\alpha^{\prime}}\left(\frac{Q_{31}^{2} u^{3}}{L^{6}}+\frac{Q_{32}^{2}}{L^{6} u^{3}}\right)\right\},
\end{aligned}
$$

up to coefficients of order unity that we suppress. Notice that the kinetic term for $b$ depends on $L$. The dependence on $L$ in the various terms in (3.8) is readily obtained from the metric (3.2), which enters via the overall volume and the inverse metric components in the contractions $\tilde{F}_{\mu_{1} \ldots \mu_{n}} G^{\mu_{1} \nu_{1}} \ldots G^{\mu_{n} \nu_{n}} \tilde{F}_{\nu_{1} \ldots \nu_{n}}$, reflecting the dilution of the fluxes at large volume.

Stabilization of $L$ (and the remainder of the moduli) will be described in a particular class of examples in section 4 below, but it is useful first to examine the axion dynamics if $L$ is imagined to be fixed, as may happen in a variety of different ways in the string landscape. The key phenomenon is that the energy built up in the $b^{4}$ term induces an adjustment of the complex structure modulus $u$, flattening the potential for $b$.

The combination in square brackets is

$$
\frac{b^{4}}{L^{8}}+\frac{b^{2}}{L^{4}}+1=\frac{\phi_{b}^{4}}{M_{P}^{4}}+\frac{\phi_{b}^{2}}{M_{P}^{2}}+1 \approx \frac{b^{4}}{L^{8}},
$$

where $\phi_{b}$ is the canonically normalized inflaton, which satisfies $\phi_{b} \gg M_{P}$ in the regime of interest for inflation. Correspondingly, we will drop the constant and quadratic terms in the square brackets from now on, in our analysis of the diagonal axion mode (3.7).

Converting to Einstein frame gives the potential

$$
V \sim M_{P}^{4} \frac{g_{s}^{4}}{L^{12}}\left(\frac{Q_{1}^{2}}{L^{4}} u b^{4}+Q_{31}^{2} u^{3}+\frac{Q_{32}^{2}}{u^{3}}\right)
$$

This potential stabilizes $u$, since it grows at large $u$ and at small $u$. For simplicity of presentation let us work in the regime where the second term in (3.10) can be neglected compared to the first and third terms. Minimizing $u$ yields the $b$-dependent vev

$$
u \approx \frac{3^{1 / 4} L}{b} \sqrt{\frac{Q_{32}}{Q_{1}}} \propto \frac{1}{b} .
$$

Substituting (3.11) into (3.10), and assuming that the kinetic energies are subleading in this dynamics, we see that the net effect is a flattening from $V \propto \phi^{4}$ to

$$
V \propto \phi^{3} .
$$


In the next two subsections we will verify that it is self-consistent to neglect kinetic energies as a source of backreaction (section 3.2), and to restrict attention to the symmetriccombination axion field $b$ in (3.7), omitting the relative coordinates $b^{(i)}-b^{(j)}, i \neq j$ (section 3.3).

Granting these facts, and anticipating a full UV completion (to be discussed in section 4), let us next estimate the scale of the parameters required for inflationary phenomenology, and check that the complex modulus $u$ is not driven to too extreme a value. A detection of $r \approx 0.1$ corresponds to (roughly)

$$
V=\frac{4}{3^{3 / 4}} \frac{g_{s}^{4}}{L^{9}} Q_{1}^{3 / 2} Q_{32}^{1 / 2} M_{P} \phi^{3} \approx 1.8 \times 10^{3} M_{P}^{4} \frac{g_{s}^{4}}{L^{9}} Q_{1}^{3 / 2} Q_{32}^{1 / 2} \sim 4 \times 10^{-9} M_{P}^{4},
$$

where we folded in the super-Planckian regime $\phi \sim 10 M_{P}$ applicable to the early period of inflation. This is straightforward to match with a moderately weak string coupling $g_{s} \lesssim 1 / 10$ and moderately large compactification radius $L \gtrsim 10$, depending on the size of the fluxes $Q_{1}, Q_{32}$. For such values of $L$ and $g_{s}$ and fluxes of order unity one finds that during inflation the mass of the canonically normalized $u$ field is larger than the Hubble scale.

Finally, let us check that we do not require such an extreme value of $u=L_{2} / L_{1}$ that new degrees of freedom appear. In particular, if $L_{2}$ were too small $\left(L_{2} \lesssim 1\right.$, corresponding to a length $L_{2} \sqrt{\alpha^{\prime}}$ below the string scale), this would lead to light winding modes; we will avoid this regime. In a more generic situation with a curved manifold, such as those we will study in section 4 , we require a large radius of curvature. Note that this does not in general require 1-cycle sizes to be larger than string scale; in the Riemann surface examples of section $4, u$ will be a complex structure modulus that does not change the curvature radius $\sim \sqrt{L_{1} L_{2} \alpha^{\prime}}$.

The mass squared of the winding modes depends on the spin structure of the circle: if fermions are anti-periodic, there is an unstable mode (winding tachyon) for radii near the string scale, whereas for a periodic spin structure there is no such instability. In either case our model is safe from winding string effects, as follows. Let us write (3.11) as

$$
u \sim \frac{L_{2}}{L_{1}} \sim \frac{M_{P}}{\phi} \frac{1}{L} \sqrt{\frac{Q_{32}}{Q_{1}}} \gtrsim 10^{-1} \frac{1}{L} \sqrt{\frac{Q_{32}}{Q_{1}}} .
$$

In (3.13) we found $L=\sqrt{L_{1} L_{2}} \gtrsim 10$, with this inequality saturated for flux quantum numbers of order 1 . In that case, (3.13) and (3.14) would be satisfied by $L_{1} \sim 10^{2}, L_{2} \sim 1$. This is already safe, and can be relaxed further (to larger $L_{2}$ ) using the fluxes $Q_{1}, Q_{32}$, allowing us to avoid extreme values of the complex structure.

\subsection{Kinetic energies}

That the kinetic energies of the axions and moduli are negligible here can be seen as follows. First, the axion kinetic term depends on the size modulus $L$, which we have temporarily assumed to be stabilized independently. Let us take the terms in the scalar potential that stabilize $L$ to be at least as large as the inflationary potential energy, and to be perturbative 
in $1 / L$. The explicit examples in the next section will satisfy this criterion. The next step is to note that during inflation, the axion kinetic term is smaller than this inflationary potential by a factor of $\varepsilon=\frac{\dot{\phi}^{2}}{H^{2} M_{P}^{2}}$. So the axion kinetic energy is a subleading source in the equation of motion for $L$.

It is likewise easy to show that the kinetic energy $u$ is also subleading in the dynamics, even though $u$ evolves during inflation, being yoked to the axion $b$ by (3.11). We can write the $u$ degree of freedom in terms of the corresponding canonically normalized scalar field $\nu$ :

$$
u \equiv e^{c_{\nu} \nu / M_{P}},
$$

where $c_{\nu}$ is a constant of order 1 . The equation of motion for $\nu$ is

$$
\ddot{\nu}+3 H \dot{\nu}=-\partial_{\nu} V .
$$

In (3.11) above, we approximated the solution to (3.16) by setting the right hand side of (3.16) to zero, by balancing two individual terms in the potential against each other. An individual term $V_{(i)}$ on the right hand side is of order

$$
\partial_{\nu} V_{(i)} \sim \frac{V_{(i)}}{M_{P}} \sim H^{2} M_{P} \sim \frac{V}{M_{P}}
$$

We will see shortly that each term on the left hand side of (3.16) is much smaller than $V / M_{P}$, so that it was indeed a good approximation to solve for the dynamics of $u=e^{c_{\nu} \nu / M_{P}}$ by setting $\partial_{\nu} V=0$.

By differentiating the relation (3.11), we see that

$$
\dot{\nu} \sim M_{P} \frac{\dot{b}}{b} \sim M_{P} \frac{\dot{\phi}}{\phi}
$$

while $\ddot{\nu}$ has terms of order $M_{P} \ddot{\phi} / \phi$ and $M_{P} \dot{\phi}^{2} / \phi^{2}$. We have

$$
H \dot{\nu} \sim M_{P} H \frac{\dot{\phi}}{\phi} \sim \sqrt{\varepsilon} \frac{M_{P}}{\phi} \frac{V}{M_{P}} \ll \partial_{\nu} V_{(i)}
$$

where in the last step we used (3.17). Similarly,

$$
M_{P} \frac{\ddot{\phi}}{\phi} \ll \frac{M_{P}}{\phi} \partial_{\phi} V \sim\left(\frac{M_{P}}{\phi}\right)^{2} \frac{V}{M_{P}} \sim\left(\frac{M_{P}}{\phi}\right)^{2} \partial_{\nu} V_{(i)} \ll \partial_{\nu} V_{(i)},
$$

where we used the fact that in slow roll inflation, $\ddot{\phi} \ll \partial_{\phi} V \sim V / \phi \sim\left(M_{P} / \phi\right)\left(V / M_{P}\right)$. Finally, the remaining contribution to $\ddot{\nu}$ is small:

$$
M_{P} \frac{\dot{\phi}^{2}}{\phi^{2}} \sim H^{2} M_{P} \times \varepsilon \frac{M_{P}^{2}}{\phi^{2}} \ll \partial_{\nu} V_{(i)}
$$

In sum, the kinetic energies are all subleading in the dynamics. 


\subsection{Transverse axion directions}

Next, let us analyze the 'relative' axion directions $b^{(i)}-b^{(j)}, i \neq j$, transverse to the configuration (3.7). These transverse directions break a symmetry, and are guaranteed to lie at an extremum of the potential. A positive mass squared in that direction, or a negative mass squared with $\left|m^{2}\right| \ll H^{2}$, does not represent an instability as we will see.

Let us focus on a given pair that contributes to $F_{1} \wedge B \wedge B$, say $b^{(1)}$ and $b^{(2)}$. Writing $b_{ \pm}=b^{(1)} \pm b^{(2)}$ and similarly for the canonical fields $\phi_{ \pm}$, the relevant terms in the potential are

$$
V \sim \lambda(u)\left(\frac{\phi_{+}^{2}}{M_{P}^{2}}+\frac{\phi_{-}^{2}}{M_{P}^{2}}+\frac{\phi_{+}^{4}}{M_{P}^{4}}+\frac{\phi_{-}^{4}}{M_{P}^{4}}-\frac{\phi_{-}^{2}}{M_{P}^{2}} \frac{\phi_{+}^{2}}{M_{P}^{2}}\right) .
$$

Here we have allowed for dependence on $u$, which in the example just discussed led to a flattening to a cubic potential for $\phi_{+}$. The last term in (3.22) introduces a negative mass squared for $\phi_{-}$that is of the same order as the positive mass squared of the perturbation $\delta \phi_{+}$. This is well below the Hubble scale at large field values: for our power law potentials,

$$
\left|m_{\delta \phi}^{2}\right| \sim \partial_{\phi}^{2} V \sim \frac{V}{\phi^{2}} \sim H^{2} \frac{M_{P}^{2}}{\phi^{2}} \ll H^{2} .
$$

There is also a subleading positive contribution from the quadratic terms (which descend from the effective $\left|F_{1} B\right|^{2}$ coupling). Depending on the details of specific models, additional positive contributions can arise, for example from the $\left|F_{3} \wedge B\right|^{2}$ term, which depends on an independent set of flux quantum numbers. In any case, even before taking into account any positive contributions, we obtain parametrically mild instabilities, $\left|m_{-}^{2}\right| \ll H^{2}$, in the transverse directions. Thus, while fluctuations of $\phi_{-}$could contribute to the primordial perturbations, instabilities in the $\phi_{-}$direction do not prevent prolonged inflation. Moreover, we do not need to sit precisely at $\phi_{-}=0$ : inflation along the $\phi_{+}$direction dominates even if we turn on $\phi_{-}$as long as $\phi_{-} \ll \phi_{+}$that ensures $\left|\partial_{\phi_{+}} V\right| \gg\left|\partial_{\phi_{-}} V\right|$.

\section{Embedding in Riemann surface compactifications}

We now turn to embedding the preceding construction in a scenario for moduli stabilization. Because the volume and string coupling will have finite masses in the stabilized vacuum, their vevs will be able to adjust to some degree, suggesting further flattening beyond that already evident in (3.12). This depends on the relative strengths of the terms in the potential that stabilize the various moduli, of which we will exhibit a few different cases.

\subsection{A concrete setup}

A natural class of compactifications to consider for this purpose is [73], for two reasons. First, the internal space (a product of Riemann surfaces) contains one-cycles that $F_{1}$ flux can thread. Secondly, this mechanism for moduli stabilization (among others) comes equipped with relatively high potential barriers against runaways to weak coupling and large radius, a feature that fits naturally with the high energy scale of large-field inflation.

This will provide additional examples realizing the general mechanism of monodromy inflation. As with previous realizations its role is to exhibit UV complete examples, and 


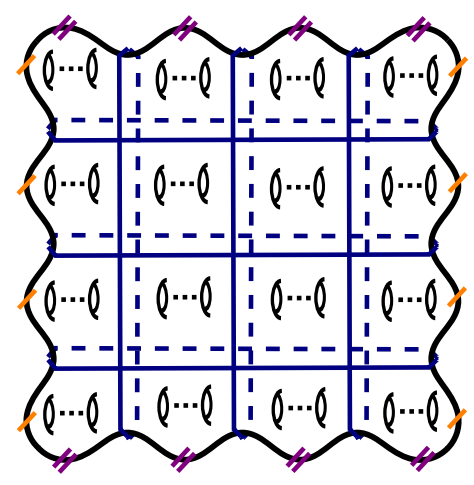

Figure 2. An example of a very symmetric Riemann surface configuration, with the loci along which various sectors of 7-branes sit marked in blue. As drawn, the 7-branes lie on contractible cycles, thereby automatically satisfying Gauss's law constraints. To create a Riemann surface with additional symmetry, we can impose periodic boundary conditions, cutting out holes where marked by single or double slashes and identifying them as indicated. In that case each 7-brane at one location needs to be balanced by an antibrane elsewhere, a configuration also consistent with the setup in [73]. The $F_{1}$ flux and legs of the $B$ field described in the text lie along the nontrivial $a$-cycles and $b$-cycles of the manifold. When microscopic consistency conditions from the orientifold projection require components of $B$ to vanish at the positions of the 7-branes, this can be achieved via suitable linear combinations as in (4.1).

in the present work an additional motivation is to explicitly map out a broader range of phenomenological predictions including the tensor to scalar ratio $r$. Any given realization is not to be taken literally, since in the string landscape there are many arbitrary choices made in choosing a total dimensionality, a compactification manifold (or generalization), fluxes, defects, and other sources. The mechanism itself - the unwinding of the potential in the presence of generic branes and fluxes - is rather robust; very specific realizations such as those developed here are meant simply as proofs of principle.

In order to incorporate axions from the Neveu-Schwarz $B$ field, we must check their compatibility with the ingredients involved in [73]. In the latter mechanism for moduli stabilization, combinations of $(p, q)$ 7-branes triply intersect — as in [72, 74] — to produce a source of negative tension scaling like that of orientifold 3-planes (O3). The resulting negative term in the four-dimensional effective potential is useful for stabilizing the Riemann surface sizes and the string coupling [73]. The coefficient of this term scales like $n_{7}^{3}$, where $n_{7}$ is the number of 7 -branes, enabling it to compete with the positive terms in the potential, including that coming from the negative curvature of the Riemann surfaces (along with the 7-brane tensions).

Let us simplify the construction [73] in the following way, preserving its essential features. Wrap the 7-branes whose triple intersections give O3 tension on homologically trivial cycles - the necks of the higher-genus Riemann surfaces as in figures 2 and 3 below. This automatically satisfies Gauss's law for all charges in the problem, and provides a symmetric, metastable configuration of these ingredients.

To be specific, as mentioned in [73] we may consider the combinations of 7-branes that behave outside their core like an O7-plane plus four D7-branes, the so-called $\mathrm{SO}(8)$ combi- 


$\left.\begin{array}{c|cc|cc|cc} & \multicolumn{2}{|c|}{\Sigma_{1}} & \multicolumn{2}{c|}{\Sigma_{2}} & \multicolumn{2}{c}{\Sigma_{3}} \\ & 1 & 2 & 3 & 4 & 5 & 6 \\ \hline \text { 7-brane } & x & & x & & x & x \\ \text { 7'-brane } & x & x & & x & x & \\ \text { 7"'-brane } & & x & x & x & & x\end{array}\right] \begin{gathered}\text { trivial } \\ \text { cycles }\end{gathered}$

Figure 3. The orientations of some of the ingredients. The 7-branes lie on the blue cycles in figure 2, while the $B$ field legs and $F_{1}$ lie on appropriate combinations of $a$ - or $b$-cycles around the handles. For example, the field $B^{(1)}$ has both legs parallel to the $7^{\prime \prime}$ sector of 7 -branes, but $B^{(1)}$ vanishes at the position of the $7^{\prime \prime}$ branes if we take a linear combination with opposite orientations around the cycles on either side of each $7^{\prime \prime}$ brane on the Riemann surface $\Sigma_{1}$ depicted in figure 2 .

nation [75]. However, we emphasize that unlike in the static, supersymmetric examples of that combination of 7-branes, in a system like ours with other forces at play, the 7-branes do not induce the asymptotic deficit angle of [75]. ${ }^{14}$

This point is worth elaboration: it is a string-theoretic version of the following standard physics. Consider $2+1$ dimensional gravity coupled to massive particles. In a static solution of the equations of motion, a massive particle induces a deficit angle proportional to its mass and the three-dimensional Newton constant $G_{N, 3}[76]$. In these static solutions, the amount of matter is bounded by $m_{\text {total }}<1 / 2 G_{N, 3}$; when one saturates this the solution becomes compact. But non-static solutions exist, including matter-dominated FRW expansion in the $2+1$ dimensional theory. In those solutions the amount of matter is not bounded: it simply determines the rate of expansion via the Friedmann equation. Similarly, 7-branes are not limited to the number - namely, 24 - that yields a static compact solution. Examples of simple time-dependent solutions involving additional 7-branes include [77, 78]. In our application, the equations of motion have contributions from various sources - the curvature, 7-branes and associated O3-planes, and fluxes. The number of 7-branes is not constrained to be 24 in this more general context.

In our compactification, we can introduce $B$ fields along cycles as indicated in the figures. In a static configuration, O7-planes would project out the constant mode of components of the $B$ fields with one leg parallel and one orthogonal to the orientifold. However, modes of this $B_{\| \perp}$ field that vanish at the O7-plane are consistent: the orientifold action essentially introduces a boundary condition that the $B$ field vanish on the fixed locus. We can satisfy this condition at the loci of the 7-branes by choosing suitable linear combinations of the $B$ fields, as explained in figure 3 .

\footnotetext{
${ }^{14}$ In the case that we wrap these on contractible cycles, we can remove the mobile D7-branes by contracting them to a point.
} 
4.2 Final powers: $p \approx 3,2,4 / 3,2 / 3, \ldots$

Finally, we are in a position to embed the example given in section 3.1 above into a compactification on a product of Riemann surfaces $\Sigma_{1} \times \Sigma_{2} \times \Sigma_{3}$. In order to describe this, let us label the $a$-cycles of $\Sigma_{1}$ collectively as $1 a$, the $b$-cycles of $\Sigma_{1}$ as $1 b$, and so on, with associated one-forms $\omega_{1 a}$, etc.

We will place $B$ fields and fluxes so as to generate a quartic term in the $B$ axions via the effective $\left|F_{1} \wedge B \wedge B\right|^{2}$ term. The potential energy described in [73] depends on complex structure moduli analogous to $u$ in section 3.1, and on the volumes of the Riemann surfaces and the string coupling $g_{s}$. Our analysis will require generalizing equation (4.7) of [73] to include the potential energy in the axions from $B$, and keeping track of the dependence of the flux potentials $n_{3}^{2}, q_{3}^{2}, q_{1}^{2}$ on the relevant complex structure modulus $\tilde{u}$ analogous to $u$ in section 3.1.

We have a variety of choices for $B$ and for $F_{1}$, as well as for the orientations of $F_{3}, H_{3}$, and $F_{5}$. To begin, let us consider a simple configuration where we place $B$ fields along two-cycles of the form

$$
B=b^{(1)} \sum_{I, J=1}^{h} \lambda_{I J} \omega_{1 b}^{I} \wedge \omega_{2 a}^{J}+b^{(2)} \sum_{I, J=1}^{h} \lambda_{I J} \omega_{2 b}^{I} \wedge \omega_{3 a}^{J}+b^{(3)} \sum_{I, J=1}^{h} \lambda_{I J} \omega_{3 b}^{I} \wedge \omega_{1 a}^{J} .
$$

This is analogous to the configuration (3.6) in section 3.1, but we orient the legs of the $B$ field as indicated so that they lie along 1-cycles on each Riemann surface factor, enabling us to enforce their vanishing at the positions of the 7-branes as described above (this is encoded in the signs $\lambda_{I J}$ ). Similarly we place $F_{1}$ flux along

$$
F_{1}=Q_{1}\left(\omega_{1 b}+\omega_{2 b}+\omega_{3 b}\right)
$$

where as we will discuss below, we either include $F_{1}$ on all such $a$ - and $b$-cycles within each Riemann surface (a maximally symmetric choice) or instead thread $F_{1}$ on a subset of these 1-cycles (or more generally, on the various cycles with different flux quantum numbers). The combination of the $B$ field (4.1) and the $F_{1}$ flux (4.2) generates a contribution to the potential energy from the effective $\left|F_{1} \wedge B \wedge B\right|^{2}$ term as in section 3.1. In this configuration, the analogue of $u$, which in this section we are calling $\tilde{u}$, is $L_{a} / L_{b}$, where $L_{a}$ and $L_{b}$ are the sizes of the $a$-cycles and $b$-cycles of the Riemann surfaces.

We will find different behavior depending on whether the $F_{5}=d C_{4}$ flux required for the stabilization construction of [73] lies along different cycles from the $F_{1} \wedge B \wedge B$ contribution to the effective $\tilde{F}_{5}$, or if instead these fluxes overlap. The latter case arises if we make the special, symmetric choice that all $h$ of the $a$ - and $b$-cycles are threaded similarly by each type of flux. The former case will provide a direct embedding of section 3.1.

At this point it is useful to introduce more of what we will need from the moduli stabilization mechanism of [73]. Although the details are specific to the particular compactifications studied there, our analysis will expose some more general lessons. The complex structure moduli of the Riemann surfaces are stabilized in [73] by a flux potential analogous to that in [72], arising from the internal components of the generalized field strengths $\tilde{F}_{p}$ 
with Lagrangian

$$
V_{(p)}=\int \sqrt{-g} \tilde{F}_{\mu_{1} \ldots \mu_{p}} g^{\mu_{1} \nu_{1}} \ldots g^{\mu_{p} \nu_{p}} \tilde{F}_{\nu_{1} \ldots \nu_{p}} .
$$

For each type of flux, (4.3) reduces to a contribution to the four-dimensional effective potential that depends on complex structure moduli, flux quantum numbers, and axions as well as on the volume and dilaton. Scaling out the latter dependencies, let us denote these flux potentials (as in [73]) as $H_{3}^{2} \rightarrow n_{3}^{2}$ and $\tilde{F}_{p}^{2} \rightarrow \tilde{q}_{p}^{2}$. We will be interested in their dependence on complex structure moduli (including the analogue of $u$ in the simple model (3.10) of section 3.1) as well as their dependence on the axions descending from $B$.

The string coupling $g_{s}$ and the volume $\mathcal{V}$ of the product of Riemann surfaces are minimized by a potential $\mathcal{U}$ that realizes a combination of 2 -term and 3 -term perturbative stabilization mechanisms (cf. e.g. [79-81]). Including the generalized fluxes $\tilde{F}_{p}$, equation (4.5) of [73] becomes

$$
\mathcal{U} \sim M_{P}^{4}\left\{\frac{h+n_{7}-1}{\sigma^{2}}-\frac{N_{7}}{\sigma^{3}}+\frac{\tilde{q}_{5}^{2}}{\sigma^{4}}+\frac{n_{3}^{2}}{\sigma^{2} \mathcal{V}^{2 / 3}}+\tilde{q}_{3}^{2} \frac{\mathcal{V}^{2 / 3}}{\sigma^{4}}+q_{1}^{2} \frac{\mathcal{V}^{4 / 3}}{\sigma^{4}}\right\}
$$

where $\sigma \equiv g_{s}^{-1} \mathcal{V}^{2 / 3}, h$ is the genus of each Riemann surface, and $n_{7}$ and $N_{7} \propto n_{7}^{3}$ are discrete parameters associated with the 7-branes in the construction [73]. This potential metastabilizes $\sigma$ with a three-term structure; in [73], the case in which the first three terms in (4.4) dominate over the others was emphasized. It is necessary for example that the three-form flux terms (i.e. those proportional to $n_{3}^{2}$ and $\tilde{q}_{3}^{2}$ ) be at least marginally subdominant, since otherwise upon integrating out $\mathcal{V}$ they produce a positive term scaling like $\sigma^{-3}$. The stabilization of $\sigma$ requires the negative term in (4.4) to be sufficiently strong. Moreover, in order for the $q_{1}^{2}$ term to at most marginally compete ${ }^{15}$ with the first three terms, we must have a hierarchy

$$
q_{1}^{2} \leq \frac{\tilde{q}_{5}^{2}}{\mathcal{V}^{4 / 3}} \ll \tilde{q}_{5}^{2}
$$

In particular, when the axion $b$ goes to zero, so that $\tilde{q}_{5} \rightarrow q_{5}$, we require $q_{1} \ll q_{5}$ at large volume $\mathcal{V}$. Given this, the flux terms $\sim n_{3}^{2}, \sim \tilde{q}_{3}^{2}$ in (4.4) stabilize the volume $\mathcal{V}$ with an essentially two-term structure, diverging at small or large $\mathcal{V}$ (for fixed $\sigma$ ). The complex structure moduli are stabilized by fluxes via a similar two-term structure encoded in the flux potential [72, 73]. Intuitively, at fixed volume and string coupling, fluxes on dual $a$-cycles and $b$-cycles cost the system increasing energy if the relative sizes of these cycles change in either direction, as then the flux becomes more concentrated.

Let us for simplicity consider 3-form fluxes that have vanishing wedge product with the $B$ fields (4.1). This is the case for the simplest generalization of (3.10) in section 3.1 to Riemann surfaces, with the three-form fluxes threading cycles consisting of products of $a$-cycles or products of $b$-cycles, as in (3.3). Then we will have two cases of interest,

\footnotetext{
${ }^{15}$ The $q_{1}^{2}$ term need not be completely subdominant (the regime studied for simplicity in [73]). It would consistent to let the $q_{1}^{2}$ term be large enough that it combined with the $n_{3}^{2}$ terms stabilizes $\mathcal{V}$, leading to a positive term scaling like $\sigma^{-8 / 3}$ which combines with the second and third terms in (4.4) to stabilize $\sigma$. In any case the $q_{1}^{2}$ term is at most marginally competitive with the leading terms, leading to (4.5).
} 
depending on whether $\left(F_{1} \wedge B \wedge B\right) \wedge d C_{4}$ is nonzero; this gives two different behaviors for the axion-dependence in $\tilde{q}_{5}^{2}$. Somewhat schematically,

$$
\begin{aligned}
& \tilde{q}_{5}^{2} \sim q_{5}^{2}+2 q_{5} q_{1} b^{2}+q_{1}^{2} b^{4} \quad \text { overlapping case (i), } \\
& \tilde{q}_{5}^{2} \sim q_{5}^{2}+q_{1}^{2} b^{4} \quad \text { non-overlapping case (ii), }
\end{aligned}
$$

where these terms depend implicitly on the complex structure moduli in a way that depends on the 1-cycles they thread (as we will make explicit below in specific examples). The canonical field is ${ }^{16} \phi \sim b M_{P} / L^{2} \sim b M_{P} / \mathcal{V}^{1 / 3}$ (for curvature radius $L \sqrt{\alpha^{\prime}}$ ), but it is useful at least at first to analyze the action in terms of $b$, while making sure to treat the $\mathcal{V}$ dependence in the kinetic term consistently.

Before moving to the complete models realizing the complex structure flattening mechanism in section 3.1, which will arise in case (ii) of (4.6), let us begin with case (i). This arises from the most symmetric choice we can make, with the fluxes threading cycles in all the handles of the Riemann surface democratically. For this first class of examples, we will keep the complex structure moduli stabilized as in [73], and focus on the dependence on $g_{s}$ and $\mathcal{V}$ (equivalently, the dependence on $\sigma$ and $\mathcal{V}$ ). To implement this, we can add the analogue of (4.1) and (4.2) in which we exchange the $a$ - and $b$-cycles, and also consider an arrangement of $F_{3}, H_{3}$, and $F_{5}$ that is symmetric under this exchange. This stabilizes the ratios of $a$ - and $b$-cycle sizes (the complex structure moduli) at a value of $\tilde{u} \sim L_{a} / L_{b}$ of order 1. (The symmetry is not essential here; more generally one can just choose $F_{1}$ and the other fluxes so that the inflationary potential depending on $b$ and the pure flux terms agree on the minimum in the $\tilde{u}$ direction.)

Given this, the volume, dilaton, and axion dynamics works as follows for case (i). We may first minimize $\sigma$ at its minimum $\sigma_{\min }$ determined by the (dominant) first three terms in (4.4), with $\tilde{q}_{5} \approx q_{5}$. Then the final step of volume and dilaton stabilization, in the presence of the axion (4.1), simply requires generalizing equation (4.7) from [73] in the following way: defining

$$
C_{h} \equiv \frac{h-n_{7}-1}{N_{7}}
$$

and incorporating the quartic term in the axion field $b=b^{(i)}, i=1,2,3$, we obtain

$$
\left.\mathcal{U}\right|_{\sigma=\sigma_{\min }} \sim M_{P}^{4}\left\{C_{h}^{2} n_{3}^{2} \frac{1}{\mathcal{V}^{2 / 3}}+C_{h}^{4}\left[q_{3}^{2}+q_{1}^{2} b^{2}\right] \mathcal{V}^{2 / 3}+C_{h}^{4}\left(q_{5}^{2}+2 q_{5} q_{1} b^{2}+q_{1}^{2} b^{4}\right)\right\} \quad \text { case (i) }
$$

valid as long as $2 q_{5} q_{1} b^{2}+q_{1}^{2} b^{4} \leq \mathcal{O}\left(q_{5}^{2}\right)$. The first two terms here stabilize the volume $\mathcal{V}$.

First, let us consider the case where $q_{3}^{2} \gg q_{1}^{2} b^{2}$. Since in the moduli stabilization mechanism [73] we have $q_{1} \ll q_{5}$, cf. (4.5), there is a window in which the inflationary potential is quadratic plus quartic in the axion, over many underlying periods of $b$. A super-Planckian vev for the canonically normalized field $\phi_{b} \sim \sqrt{h} M_{P} b / \mathcal{V}^{1 / 3}$ (with $h$ the genus of the Riemann surfaces), keeping the axion terms at most marginally competitive with the $q_{5}^{2}$ term in (4.8), requires

$$
\frac{q_{1} \mathcal{V}^{2 / 3}}{h q_{5}} \leq \frac{M_{P}^{2}}{\phi^{2}}
$$

\footnotetext{
${ }^{16}$ This expression omits possible dependence on the genus $h$.
} 
which is satisfied given a moderately large $h$ (as is required to be able to tune the cosmological constant in [73]) or given a significant hierarchy between $q_{1}$ and $q_{5}$. We impose (4.9) because if the axion terms dominated over the $q_{5}^{2}$ term, then the third term in (4.4) would vary over several orders of magnitude as $\phi_{b}$ varies from $\sim 10 M_{P}$ to $\sim M_{P}$, which would invalidate the three-term stabilization of $\sigma$. The condition (4.9) in turn ensures that the quadratic term in the round brackets of (4.8) dominates over the quartic term in the axion, although the latter could become significant at the outer edge of the super-Planckian regime. (This latter effect may be interesting in light of the hints of a tension between Planck and BICEP2 [82-85], although that tension is highly uncertain given [86, 87] as well as foreground unknowns.)

Since we have expressed the action in terms of $b$ rather than the canonical field $\phi \sim$ $\sqrt{h} M_{P} b / \mathcal{V}^{1 / 3}$, we must take into account the $\mathcal{V}$ dependence in the kinetic term for $b$, $\mathcal{S}_{\text {kin }} \sim \int M_{P}^{2} h \dot{b}^{2} / \mathcal{V}^{2 / 3}$. However, this is easily subdominant in the dynamics of $\mathcal{V}$, since the inflaton kinetic energy is much less than its potential energy (by a factor of the inflationary slow roll parameter $\varepsilon$ ), and this in turn may be easily kept smaller than each of the first two terms in (4.8). This last statement follows from the subdominance of the $q_{1}$ contribution to the moduli potential in [73]. Altogether, from case (i) of (4.6) we have obtained a quadratic inflaton potential, crossing over to quartic at the boundary of its large field range (as it reaches the regime where the inflaton potential would destabilize the modulus $\sigma$ ).

In more general circumstances, as we will see momentarily in a specific example, the kinetic term can play a more nontrivial role in the dynamics; if $\mathcal{V}$ depends on $b$, this affects the definition of the canonically normalized inflaton field $\phi_{b}$, which can alter the ultimate power of the potential [30]. In the special case that the kinetic term after volume stabilization is proportional to $(\dot{b} / b)^{2}$, this change can prevent inflation, as it renders a potential that is power law in $b$ exponential in terms of the canonically normalized field. Without a sufficiently small coefficient in the exponent (which may arise in some cases, but not generally), or a separation of mass scales, this will not inflate.

Next, let us consider the case where $q_{1}^{2} b^{2} \gg q_{3}^{2}$ in the square brackets in (4.8). In this case, we obtain $b \propto 1 / \mathcal{V}^{2 / 3}$ from the first two terms in the potential (4.8). This has two effects: it introduces a linear term in $b$ in the potential, and it also changes the relation between $b$ and the canonically normalized inflaton field $\phi_{b}$ because of the $\mathcal{V}$ dependence in the $b$ kinetic term

$$
\frac{\dot{b}^{2}}{\mathcal{V}^{2 / 3}} \propto \dot{b}^{2} b \Rightarrow \phi_{b} \propto b^{3 / 2}
$$

(see [30] for previous examples of this effect). Given (4.10), for the regime (4.9) in which the quadratic term in $b \propto q_{1} q_{5} b^{2}$ dominates in the potential, one finds $p=(2 / 3) \times 2=4 / 3$ :

$$
V\left(\phi_{b}\right) \approx \mu^{8 / 3} \phi^{4 / 3}
$$

Before moving on to complex structure adjustments, we can obtain another class of models from the case $p_{0}=2$. Consider a set of $B$ fields for which $B \wedge B$ vanishes (or is negligible), obtainable by appropriate distribution of the legs of $B$ among the handles of the Riemann surfaces. As above, we take the dominant flux terms - including $q_{5}$ - to stabilize the corresponding complex structure moduli, as in [73]. The field configuration (2.8) gives 
a contribution to $F_{3}$ of the form $F_{1} \wedge B$, orthogonal to the components of $F_{3}$ we prescribed above (in which the three legs are either all on $a$-cycles or all on $b$-cycles). In the absence of $B \wedge B$ contributions, the potential takes the form

$$
\left.\mathcal{U}\right|_{\sigma=\sigma_{\min }} \sim M_{P}^{4}\left\{C_{h}^{2} n_{3}^{2} \frac{1}{\mathcal{V}^{2 / 3}}+C_{h}^{4}\left(q_{3}^{2}+q_{1}^{2} b^{2}\right) \mathcal{V}^{2 / 3}+C_{h}^{4} q_{5}^{2}\right\} \quad \text { case (i) }
$$

The result is a linear contribution to the potential in $b$, as in the previous example, but here there are no additional quadratic or quartic terms. The kinetic term works as in (4.10), giving $p=2 / 3$ :

$$
V \approx \mu^{10 / 3} \phi^{2 / 3}
$$

Let us next move to case (ii) of (4.6), which gives an a priori quartic dependence on $b$, i.e. a fiducial power $p_{0}=4$. In these next examples, we will also incorporate a more general complex structure dependence, including dependence on a modulus $\tilde{u}=L_{a} / L_{b}$ describing the ratio of $a$ - and $b$-cycle sizes in some subset of Riemann surface handles.

$$
\left.\mathcal{U}\right|_{\sigma=\sigma_{\min }} \sim M_{P}^{4}\left\{C_{h}^{2} n_{3}(\tilde{u})^{2} \frac{1}{\mathcal{V}^{2 / 3}}+C_{h}^{4} q_{3}(\tilde{u})^{2} \mathcal{V}^{2 / 3}+C_{h}^{4}\left[q_{5}(\tilde{u})^{2}+q_{1}(\tilde{u})^{2} b^{4}\right]\right\} \quad \text { case (ii) . }
$$

The $\tilde{u}$ dependence arises from the dependence of the flux energies on the complex structure derived explicitly in [73], along with the analogous complex structure dependence in the axion potential terms (coming from the $B$ dependence in the generalized fluxes $\tilde{F}_{p}$ ). Depending on how we distribute the legs of the fluxes, each type of flux that threads 1-cycles within the Riemann surfaces can depend on $\tilde{u} \sim L_{b} / L_{a}$ as a combination of terms of order $\tilde{u}^{ \pm 1}$, and for the three-forms $H_{3}$ and $F_{3}$ we can also have terms of order $\tilde{u}^{ \pm 3}$ from fluxes threading a one-cycle of each of the three Riemann surfaces (as in the model (3.10) in section 3.1). Our two-form potential $B$ threads two-cycles composed of a product of $a$ - and $b$-cycles (4.1), and so $b$ does not have any implicit $\tilde{u}$ dependence.

To obtain more general examples, we can break some of the symmetry assumed in the first set of examples described above. There are two ways in which we can generalize: (I) break the symmetry among the different pairs of $a$ - and b-cycles on each Riemann surface, and/or (II) break the symmetry among the three Riemann surfaces. We will next consider two sets of examples, in the first case relaxing the symmetry just in sense (I) and in the second set generalizing in the direction of both (I) and (II) together. This will give us powers $p \approx 3$ and $p \approx 2$ respectively, starting from the fiducial power $p_{0}=4$.

First, let us consider particular subsets of pairs of $a$-cycles and $b$-cycles on which to thread the $F_{1}$ flux, treating the handles of each Riemann surface less symmetrically. In this case, the inflaton potential term $V \propto q_{1}^{2} b^{p_{0}}$ with $p_{0}=4$ has a distinct dependence on the corresponding complex structure moduli $\tilde{u}_{I}=L_{a I} / L_{b I}$ (where the index $I=1, \ldots, n_{1}$ runs over the handles threaded by $F_{1}$ - taking at least the minimal number required to respect the consistency conditions from the 7-branes). Let us also separate the fluxes that stabilize the complex structure moduli in [73] into those with legs on these $n_{1}$ cycles (which we can label $\Delta F_{p}$ ) and those without legs on them (which we will call $F_{p}^{(0)}$ ). The latter we can take to dominate in stabilizing the string coupling and volume in (4.4), which proceeds as 
described in [73]. (There $F_{1}^{(0)}$ is not required, and we can consider for simplicity $F_{1}=\Delta F_{1}$, i.e. only threading $F_{1}$ on $n_{1}$ of the cycles as just prescribed.)

The set of fluxes threading cycles within the $n_{1}$ handles, i.e. the $\Delta F_{p}$ fluxes, includes some that depend on the combination $L_{a I} L_{b I}$, and others that depend on the ratio $\tilde{u}_{I}=$ $L_{a I} / L_{b I}$. The former combined with the $F_{P}^{(0)}$ fluxes stabilize the product $L_{a I} L_{b I}$ as in [73], provided that one chooses large enough flux quantum numbers in these sectors so that this is a leading effect.

Finally, we can address the stabilization of $\tilde{u}_{I}=L_{a I} / L_{b I}$. The $B$ fields and $F_{1}$, and the remaining $\Delta F_{p}$ fluxes stabilize this just as in the model (3.4), (3.6), and (3.10) explained in section 3.1 (replacing $u$ in that toroidal toy model with $\tilde{u}$ in the Riemann surface compactification). The $b$ kinetic term depends only on the volume of a given handle (the product $L_{a I} L_{b I}$ ), not on $\tilde{u} \sim L_{a I} / L_{b I}$. At the minimum in the $\sigma$ and $\mathcal{V}$ directions, the $\tilde{u}$ dependence in the potential is of the form (cf. (3.10))

$$
\left.\mathcal{U}\right|_{*} \sim M_{P}^{4} C_{h}^{2}\left\{\left(\frac{\Delta n_{31}^{2}}{\mathcal{V}_{*}^{2 / 3}}+\Delta q_{31}^{2} \mathcal{V}_{*}^{2 / 3}\right) \tilde{u}^{3}+\left(\frac{\Delta n_{32}^{2}}{\mathcal{V}_{*}^{2 / 3}}+\Delta q_{32}^{2} \mathcal{V}_{*}^{2 / 3}\right) \frac{1}{\tilde{u}^{3}}+C_{h}^{2} q_{1}^{2} b^{4} \tilde{u}\right\}
$$

where $\left.\mathcal{U}\right|_{*}$ is shorthand for $\left.\mathcal{U}\right|_{\sigma=\sigma_{*}, \mathcal{V}=\mathcal{V}_{*}}$, and we stress that (4.15) applies in case (ii).

As in the previous example, we work in the regime where the axion kinetic term is a subdominant source in the equation of motion for $L_{a I} L_{b I}$, leaving $L_{a I} L_{b I}$ stabilized as in [73]. The kinetic term for $\tilde{u}$ is also subleading in the dynamics, as explained above in section 3.2. Altogether, stabilizing $\tilde{u}$ during inflation using the last two terms in (4.15), as in section 3.1 this gives a flattening to a cubic potential from the fiducial quartic potential,

$$
V \approx \mu \phi^{3}, \quad p_{0}=4 \rightarrow p=3 .
$$

For another class of examples, we can relax the symmetry further and allow the three Riemann surface factors to behave differently. Then, instead of a cubic dependence on $\tilde{u}$ in the three-form flux terms, we obtain $\tilde{u}^{ \pm 1}$. This, combined with the dependence $b^{4} \tilde{u}$ in the axion term, leads to $\tilde{u} \sim 1 / b^{2}$, and $V \propto b^{2}$. That is, this last class of examples produces to good approximation $\frac{1}{2} m^{2} \phi^{2}$ inflation,

$$
V \approx \frac{1}{2} m^{2} \phi^{2}, \quad p_{0}=4 \rightarrow p=2 .
$$

It is clear from the examples considered thus far that various powers appear, giving a wide range of (discretely different) values of $r$. A quadratic potential is among them, coming either as the result of rigid stabilization with a quadratic potential, or via flattening from a quartic potential. However, from the top down the quadratic model is not particularly special. It is a classic model from the bottom up [23], and is simple in some sense. But this simplicity may be illusory - the field theory model alone does not account for quantum gravity effects (or particle physics or the cosmological constant). From the top down, the monodromy mechanism for large fields that underlies this and other examples appears to be quite simple, with moduli stabilization introducing what complications there are in the problem. As we have seen here, the inflationary dynamics itself can participate in a rather simple way in moduli stabilization, simplifying the latter somewhat. 


\section{Monodromies of complex structure moduli}

For special classes of compactification manifolds, such as Calabi-Yau spaces and Riemann surfaces, the metric deformations include complex structure moduli. Their monodromies play an important role in the mathematical structure of these compactifications, particularly in the Calabi-Yau case where much of the moduli space geometry has been mapped out. As mentioned above, these generalize the $\tau \rightarrow \tau+1$ symmetry for a torus, for which $\operatorname{Re}(\tau)$ plays the role of an axion.

It is natural to consider these moduli as candidate inflatons, a topic we leave mainly for future work. But as a start, it is straightforward to derive a close analogue of the examples given in section 3.1, starting from the T-duality between $B$ fields and angular metric deformations. Specifically, we T-dualize on the $y_{2}$ directions of the $T^{2}$ factors in that toy model. For each torus, T-duality exchanges

$$
\rho=b+i \sqrt{G} \leftrightarrow \quad \tau=\frac{G_{12}}{G_{22}}+i \frac{\sqrt{G}}{G_{22}} \equiv \tau_{1}+i \tau_{2},
$$

where the metric of the $T^{2}$ is $d s^{2}=G_{M N} d y^{M} d y^{N}$ and $\sqrt{G}=L^{2}$ is the volume. The quasiperiodic direction under $b \rightarrow b+1$ maps to $\tau \rightarrow \tau+1$. The effective flux coupling $\left|F_{1} \wedge B \wedge B\right|^{2}$ yielded the monodromy-induced quartic coupling in (3.10). Under the Tduality (5.1), $F_{1}$ dualizes to four-form flux $F_{4}$, and and the three-form fluxes dualize to $F_{0}$ and $F_{6}$. The resulting effective potential on the T-dual side is, for $\tau_{1} \gg 1$,

$$
V \sim M_{P}^{4} \frac{g_{s}^{4}}{L^{12}}\left(Q_{4}^{2} \frac{\tau_{1}^{4}}{\rho_{2} \tau_{2}^{2}}+\rho_{2}^{3} Q_{0}^{2}+\frac{Q_{6}^{2}}{\rho_{2}^{3}}\right)
$$

as can be computed directly using the T-dual fluxes and metric, or by applying (5.1) to (3.10). In parallel to the previous case, solving for $\rho_{2}$ here gives a cubic inflationary potential along the $\tau_{1}$ direction. We leave the study of generalizations that are not directly T-dual to previous examples as an interesting problem for the future.

\section{Conclusions}

Monodromies of axion fields are ubiquitous in string compactifications with sufficiently general fluxes or brane configurations. In this work we first provided an overview of the monodromy mechanism, emphasizing the genericity of the large field ranges induced by flux couplings along axion directions, as well as the role of the underlying discrete shift symmetry in protecting other aspects of the physics. Just as the potential exhibits a branch structure with an underlying periodicity, as in figure 1, there is a periodicity in the spectrum of branes wrapping the cycles that yield axions from higher-dimensional potential fields. Inflation proceeds on one branch of the monodromy-extended potential, while these sectors of the spectrum remain periodic.

While it is straightforward to identify compactifications containing fields and couplings that appear suitable for large-field inflation, stabilizing moduli remains the primary technical complication, both in monodromy constructions and in all other scenarios for inflation 
in string theory. The most detailed and explicit scenario presented in this work builds on the construction of [73], in which the moduli of type IIB string theory compactified on a product of Riemann surfaces are stabilized by fluxes and $(p, q)$ 7-branes. In this setting, the inflaton corresponds to an axion descending from the NS-NS two-form $B$, and the monodromy is a consequence of the coupling $\left|F_{1} \wedge B \wedge B\right|^{2}$ T-dual to the coupling $\left|m_{0} B \wedge B\right|^{2}$ in massive type IIA string theory. We also related this via T-duality to monodromies in complex structure moduli space, which may provide another rich set of examples to explore; constructing more explicit and general examples along those lines is an important task for the future.

We presented several new models of large-field inflation from axion monodromy, involving monomial potentials $\mu^{4-p} \phi^{p}$. A key phenomenon is flattening [30], in which the inflationary potential energy density makes a leading contribution to the potential for some of the moduli, whose vevs then adjust during the course of inflation, reducing the total energy. The result is that an exponent $p_{0}$ computed in the absence of flattening is reduced to $p<p_{0}$ by the dynamical adjustment of the moduli. In this work, we exhibited examples with $p=3,2,4 / 3$, and $2 / 3$, realizing a large range of phenomenological predictions for the tensor to scalar ratio. This includes a class of examples with flattening from $p_{0}=4$ to $p=3$, somewhat analogous to the flattening from $p_{0}=2$ to $p=1$ in $[25,26,30]$. It would be extremely interesting to build from this experience to more systematically characterize the powers arising in monodromy inflation. The present work, as well as [30, 35], provide a modest start to this program, by incorporating the natural interplay between inflation and moduli stabilization.

The monodromy structure of string theory axions, and their duals among complex structure moduli and brane positions, has played an interesting mathematical role in the theory, and naturally generates large-field inflation. Phenomenologically, the discrete examples of $p$ obtained in this and other works, and a more systematic generalization if that can be accomplished, relate directly to various cosmological observables. It is of great interest to understand the spectrum of UV-complete values of $r$ (a detectable amplitude of tensor fluctuations being the main model-independent signature of monodromy inflation) as well as $n_{s}$ (which depends on $p$ and also on the number of fields involved [36-47]). In addition, one would like to map out the more detailed, but model-dependent signatures from the residual oscillations in the potential $(1.1)[25,26]$ generated by the sectors of the physics that respect the underlying periodicity $\phi \rightarrow \phi+2 \pi f$. The search for such oscillations — which has so far led to constraints [88-94] — may be affected by the theoretical spectrum of possible values of $p$, and by the possibility of dynamical relaxation of the period $f$ and of the model-dependent amplitude $\Lambda^{4}$ of the oscillations during inflation. All this provides ample motivation for further study.

\section{Acknowledgments}

We thank X. Dong, S. Hartnoll, S. Kachru, R. Kallosh, N. Kaloper, A. Lawrence, A. Linde, J. Maldacena, P. McGuirk, J. Polchinski, L. Senatore, S. Shenker, G. Torroba, E. Witten, and M. Zaldarriaga for useful discussions. The research of L.M. was supported by NSF 
grant PHY-0757868. The work of E.S. was supported in part by the National Science Foundation under grant PHY-0756174 and NSF PHY11-25915 and by the Department of Energy under contract DE-AC03-76SF00515. The research of A.W. was supported by the Impuls und Vernetzungsfond of the Helmholtz Association of German Research Centres under grant HZ-NG-603. A.W. would like to thank SITP, where the main part of this work was completed, for their extraordinarily warm hospitality. T.W. was supported by a Research Fellowship (Grant number WR 166/1-1) of the German Research Foundation (DFG).

Open Access. This article is distributed under the terms of the Creative Commons Attribution License (CC-BY 4.0), which permits any use, distribution and reproduction in any medium, provided the original author(s) and source are credited.

\section{References}

[1] N. Seiberg, The power of holomorphy: exact results in $4 D$ SUSY field theories, hep-th/9408013 [INSPIRE].

[2] A.H. Guth, The inflationary universe: a possible solution to the horizon and flatness problems, Phys. Rev. D 23 (1981) 347 [InSPIRE].

[3] A.D. Linde, A new inflationary universe scenario: a possible solution of the horizon, flatness, homogeneity, isotropy and primordial monopole problems, Phys. Lett. B 108 (1982) 389 [INSPIRE].

[4] A. Albrecht and P.J. Steinhardt, Cosmology for grand unified theories with radiatively induced symmetry breaking, Phys. Rev. Lett. 48 (1982) 1220 [INSPIRE].

[5] A.A. Starobinsky, A new type of isotropic cosmological models without singularity, Phys. Lett. B 91 (1980) 99 [inSPIRE].

[6] V.F. Mukhanov and G.V. Chibisov, Quantum fluctuation and nonsingular universe (in Russian), JETP Lett. 33 (1981) 532 [Pisma Zh. Eksp. Teor. Fiz. 33 (1981) 549] [InSPIRE].

[7] G.V. Chibisov and V.F. Mukhanov, Galaxy formation and phonons, Mon. Not. Roy. Astron. Soc. 200 (1982) 535 [INSPIRE].

[8] A.H. Guth and S.Y. Pi, Fluctuations in the new inflationary universe, Phys. Rev. Lett. 49 (1982) 1110 [INSPIRE].

[9] S.W. Hawking, The development of irregularities in a single bubble inflationary universe, Phys. Lett. B 115 (1982) 295 [INSPIRE].

[10] A.A. Starobinsky, Dynamics of phase transition in the new inflationary universe scenario and generation of perturbations, Phys. Lett. B 117 (1982) 175 [InSPIRE].

[11] A.A. Starobinsky, The perturbation spectrum evolving from a nonsingular initially de-Sitter cosmology and the microwave background anisotropy, Sov. Astron. Lett. 9 (1983) 302 [INSPIRE].

[12] J.M. Bardeen, J.R. Bond, N. Kaiser and A.S. Szalay, The statistics of peaks of Gaussian random fields, Astrophys. J. 304 (1986) 15 [INSPIRE].

[13] K.N. Abazajian et al., Inflation physics from the cosmic microwave background and large scale structure, arXiv:1309.5381 [INSPIRE]. 
[14] D.H. Lyth, What would we learn by detecting a gravitational wave signal in the cosmic microwave background anisotropy?, Phys. Rev. Lett. 78 (1997) 1861 [hep-ph/9606387] [INSPIRE].

[15] G. Efstathiou and K.J. Mack, The Lyth bound revisited, JCAP 05 (2005) 008 [astro-ph/0503360] [INSPIRE].

[16] M. Farhang, J.R. Bond, O. Doré and C.B. Netterfield, Primordial gravitational wave detectability with deep small-sky cosmic microwave background experiments, Astrophys. J. 771 (2013) 12 [arXiv:1108.2043] [INSPIRE].

[17] BICEP2 collaboration, P.A.R. Ade et al., Detection of B-mode polarization at degree angular scales by BICEP2, Phys. Rev. Lett. 112 (2014) 241101 [arXiv:1403.3985] [INSPIRE].

[18] BICEP2 collaboration, P.A.R. Ade et al., BICEP2 II: experiment and three-year data set, Astrophys. J. 792 (2014) 62 [arXiv:1403.4302] [InSPIRE].

[19] U. Seljak and M. Zaldarriaga, Signature of gravity waves in polarization of the microwave background, Phys. Rev. Lett. 78 (1997) 2054 [astro-ph/9609169] [INSPIRE].

[20] M. Kamionkowski, A. Kosowsky and A. Stebbins, A probe of primordial gravity waves and vorticity, Phys. Rev. Lett. 78 (1997) 2058 [astro-ph/9609132] [INSPIRE].

[21] Planck collaboration, P.A.R. Ade et al., Planck intermediate results. XIX. An overview of the polarized thermal emission from galactic dust, arXiv:1405.0871 [INSPIRE].

[22] PLANCK collaboration, P.A.R. Ade et al., Planck intermediate results. XXII. Frequency dependence of thermal emission from galactic dust in intensity and polarization, arXiv: 1405.0874 [INSPIRE].

[23] A.D. Linde, Chaotic inflation, Phys. Lett. B 129 (1983) 177 [InSPIRE].

[24] E. Silverstein and A. Westphal, Monodromy in the CMB: gravity waves and string inflation, Phys. Rev. D 78 (2008) 106003 [arXiv:0803.3085] [INSPIRE].

[25] L. McAllister, E. Silverstein and A. Westphal, Gravity waves and linear inflation from axion monodromy, Phys. Rev. D 82 (2010) 046003 [arXiv: 0808.0706] [InSPIRE].

[26] R. Flauger, L. McAllister, E. Pajer, A. Westphal and G. Xu, Oscillations in the CMB from axion monodromy inflation, JCAP 06 (2010) 009 [arXiv:0907.2916] [INSPIRE].

[27] N. Kaloper and L. Sorbo, A natural framework for chaotic inflation, Phys. Rev. Lett. 102 (2009) 121301 [arXiv:0811.1989] [INSPIRE].

[28] N. Kaloper, A. Lawrence and L. Sorbo, An ignoble approach to large field inflation, JCAP 03 (2011) 023 [arXiv:1101.0026] [INSPIRE].

[29] N. Kaloper and A. Lawrence, Natural chaotic inflation and UV sensitivity, Phys. Rev. D 90 (2014) 023506 [arXiv:1404.2912] [INSPIRE].

[30] X. Dong, B. Horn, E. Silverstein and A. Westphal, Simple exercises to flatten your potential, Phys. Rev. D 84 (2011) 026011 [arXiv:1011.4521] [INSPIRE].

[31] S. Dubovsky, A. Lawrence and M.M. Roberts, Axion monodromy in a model of holographic gluodynamics, JHEP 02 (2012) 053 [arXiv: 1105.3740] [INSPIRE].

[32] G. D'Amico, R. Gobbetti, M. Schillo and M. Kleban, Inflation from flux cascades, Phys. Lett. B 725 (2013) 218 [arXiv:1211.3416] [INSPIRE].

[33] G. D’Amico, R. Gobbetti, M. Kleban and M. Schillo, Unwinding inflation, JCAP 03 (2013) 004 [arXiv: 1211.4589] [INSPIRE]. 
[34] B. Shlaer, Chaotic brane inflation, Phys. Rev. D 88 (2013) 103503 [arXiv:1211.4024] [INSPIRE].

[35] G. Gur-Ari, Brane inflation and moduli stabilization on twisted tori, JHEP 01 (2014) 179 [arXiv: 1310.6787] [INSPIRE].

[36] E. Palti and T. Weigand, Towards large $r$ from $[p, q]$-inflation, JHEP 04 (2014) 155 [arXiv: 1403.7507] [INSPIRE].

[37] F. Marchesano, G. Shiu and A.M. Uranga, F-term axion monodromy inflation, arXiv: 1404.3040 [INSPIRE].

[38] K. Harigaya and M. Ibe, Inflaton potential on a Riemann surface, arXiv:1404.3511 [INSPIRE].

[39] A. Hebecker, S.C. Kraus and L.T. Witkowski, D7-brane chaotic inflation, Phys. Lett. B 737 (2014) 16 [arXiv:1404.3711] [INSPIRE].

[40] L.E. Ibáñez and I. Valenzuela, The inflaton as a MSSM Higgs and open string modulus monodromy inflation, arXiv:1404.5235 [INSPIRE].

[41] T. Kobayashi, O. Seto and Y. Yamaguchi, Axion monodromy inflation with sinusoidal corrections, arXiv:1404.5518 [INSPIRE].

[42] M. Dine, P. Draper and A. Monteux, Monodromy inflation in SUSY QCD, JHEP 07 (2014) 146 [arXiv: 1405.0068] [INSPIRE].

[43] M. Arends et al., D\%-brane moduli space in axion monodromy and fluxbrane inflation, Fortschr. Phys. 62 (2014) 647 [arXiv:1405.0283] [INSPIRE].

[44] K. Yonekura, Notes on natural inflation, arXiv:1405.0734 [INSPIRE].

[45] T. Higaki, T. Kobayashi, O. Seto and Y. Yamaguchi, Axion monodromy inflation with multi-natural modulations, arXiv:1405.0775 [INSPIRE].

[46] D. Wenren, Tilt and tensor-to-scalar ratio in multifield monodromy inflation, arXiv:1405.1411 [INSPIRE].

[47] F. Hassler, D. Lüst and S. Massai, On inflation and de Sitter in non-geometric string backgrounds, arXiv:1405.2325 [INSPIRE].

[48] J.R. Bond, L. Kofman, S. Prokushkin and P.M. Vaudrevange, Roulette inflation with Kähler moduli and their axions, Phys. Rev. D 75 (2007) 123511 [hep-th/0612197] [INSPIRE].

[49] R. Blumenhagen and E. Plauschinn, Towards universal axion inflation and reheating in string theory, Phys. Lett. B 736 (2014) 482 [arXiv: 1404.3542] [INSPIRE].

[50] T.W. Grimm, Axion inflation in F-theory, arXiv: 1404.4268 [INSPIRE].

[51] K. Choi, H. Kim and S. Yun, Natural inflation with multiple sub-Planckian axions, Phys. Rev. D 90 (2014) 023545 [arXiv:1404.6209] [InSPIRE].

[52] T. Higaki and F. Takahashi, Natural and multi-natural inflation in axion landscape, JHEP 07 (2014) 074 [arXiv: 1404.6923] [INSPIRE].

[53] S.H.H. Tye and S.S.C. Wong, Helical inflation and cosmic strings, arXiv:1404.6988 [INSPIRE].

[54] R. Kappl, S. Krippendorf and H.P. Nilles, Aligned natural inflation: monodromies of two axions, Phys. Lett. B 737 (2014) 124 [arXiv:1404.7127] [InSPIRE].

[55] T.C. Bachlechner, M. Dias, J. Frazer and L. McAllister, A new angle on chaotic inflation, arXiv: 1404.7496 [INSPIRE]. 
[56] I. Ben-Dayan, F.G. Pedro and A. Westphal, Hierarchical axion inflation, arXiv:1404.7773 [INSPIRE].

[57] C. Long, L. McAllister and P. McGuirk, Aligned natural inflation in string theory, Phys. Rev. D 90 (2014) 023501 [arXiv: 1404.7852] [inSPIRE].

[58] R. Kallosh, A. Linde and A. Westphal, Chaotic inflation in supergravity after Planck and BICEP2, Phys. Rev. D 90 (2014) 023534 [arXiv:1405.0270] [INSPIRE].

[59] A. Ashoorioon and M.M. Sheikh-Jabbari, Gauged M-flation after BICEP2, arXiv:1405.1685 [INSPIRE].

[60] K. Freese, J.A. Frieman and A.V. Olinto, Natural inflation with pseudo-Nambu-Goldstone bosons, Phys. Rev. Lett. 65 (1990) 3233 [INSPIRE].

[61] F.C. Adams, J.R. Bond, K. Freese, J.A. Frieman and A.V. Olinto, Natural inflation: particle physics models, power law spectra for large scale structure and constraints from COBE, Phys. Rev. D 47 (1993) 426 [hep-ph/9207245] [INSPIRE].

[62] J.E. Kim, H.P. Nilles and M. Peloso, Completing natural inflation, JCAP 01 (2005) 005 [hep-ph/0409138] [INSPIRE].

[63] S. Dimopoulos, S. Kachru, J. McGreevy and J.G. Wacker, N-flation, JCAP 08 (2008) 003 [hep-th/0507205] [INSPIRE].

[64] T.W. Grimm, Axion inflation in type-II string theory, Phys. Rev. D 77 (2008) 126007 [arXiv: 0710.3883] [INSPIRE].

[65] J. Polchinski, String theory. Vol. 2: Superstring theory and beyond, Cambridge University Press, Cambridge U.K. (1998) [INSPIRE].

[66] M. Dodelson, X. Dong, E. Silverstein and G. Torroba, New solutions with accelerated expansion in string theory, arXiv:1310.5297 [INSPIRE].

[67] E. Bergshoeff, M. de Roo, M.B. Green, G. Papadopoulos and P.K. Townsend, Duality of type-II 7 branes and 8 branes, Nucl. Phys. B 470 (1996) 113 [hep-th/9601150] [InSPIRE].

[68] H. Ooguri and C. Vafa, On the geometry of the string landscape and the swampland, Nucl. Phys. B 766 (2007) 21 [hep-th/0605264] [INSPIRE].

[69] L. Smolin, Gravitational radiative corrections as the origin of spontaneous symmetry breaking!, Phys. Lett. B 93 (1980) 95 [INSPIRE].

[70] N.C. Bizet, A. Klemm and D.V. Lopes, Landscaping with fluxes and the E8 Yukawa point in F-theory, arXiv:1404.7645 [INSPIRE].

[71] E. Witten, Phases of $N=2$ theories in two-dimensions, Nucl. Phys. B 403 (1993) 159 [hep-th/9301042] [INSPIRE].

[72] S.B. Giddings, S. Kachru and J. Polchinski, Hierarchies from fluxes in string compactifications, Phys. Rev. D 66 (2002) 106006 [hep-th/0105097] [INSPIRE].

[73] A. Saltman and E. Silverstein, A new handle on de Sitter compactifications, JHEP 01 (2006) 139 [hep-th/0411271] [INSPIRE].

[74] S. Kachru, R. Kallosh, A.D. Linde and S.P. Trivedi, De Sitter vacua in string theory, Phys. Rev. D 68 (2003) 046005 [hep-th/0301240] [INSPIRE].

[75] A. Sen, F theory and orientifolds, Nucl. Phys. B 475 (1996) 562 [hep-th/9605150] [INSPIRE]. 
[76] S. Deser, R. Jackiw and G. 't Hooft, Three-dimensional Einstein gravity: dynamics of flat space, Annals Phys. 152 (1984) 220 [INSPIRE].

[77] M. Kleban and M. Redi, Expanding F-theory, JHEP 09 (2007) 038 [arXiv:0705.2020] [INSPIRE].

[78] X. Dong, B. Horn, S. Matsuura, E. Silverstein and G. Torroba, FRW solutions and holography from uplifted AdS/CFT, Phys. Rev. D 85 (2012) 104035 [arXiv:1108.5732] [INSPIRE].

[79] A. Maloney, E. Silverstein and A. Strominger, De Sitter space in noncritical string theory, hep-th/0205316 [INSPIRE].

[80] E. Silverstein, (A)dS backgrounds from asymmetric orientifolds, hep-th/0106209 [INSPIRE].

[81] E. Silverstein, Simple de Sitter solutions, Phys. Rev. D 77 (2008) 106006 [arXiv:0712.1196] [INSPIRE].

[82] A. Ashoorioon, K. Dimopoulos, M.M. Sheikh-Jabbari and G. Shiu, Non-Bunch-Davies initial state reconciles chaotic models with BICEP and Planck, Phys. Lett. B 737 (2014) 98 [arXiv: 1403.6099] [INSPIRE].

[83] K.M. Smith et al., On quantifying and resolving the BICEP2/Planck tension over gravitational waves, Phys. Rev. Lett. 113 (2014) 031301 [arXiv:1404.0373] [INSPIRE].

[84] B. Freivogel, M. Kleban, M.R. Martinez and L. Susskind, Observational consequences of a landscape: epilogue, arXiv: 1404.2274 [INSPIRE].

[85] R. Bousso, D. Harlow and L. Senatore, Inflation after false vacuum decay: new evidence from BICEP2, arXiv: 1404.2278 [INSPIRE].

[86] D. Spergel, R. Flauger and R. Hlozek, Planck data reconsidered, arXiv: 1312.3313 [INSPIRE].

[87] B. Audren, D.G. Figueroa and T. Tram, A note of clarification: BICEP2 and Planck are not in tension, arXiv:1405.1390 [INSPIRE].

[88] R. Easther and R. Flauger, Planck constraints on monodromy inflation, JCAP 02 (2014) 037 [arXiv: 1308.3736] [INSPIRE].

[89] H. Peiris, R. Easther and R. Flauger, Constraining monodromy inflation, JCAP 09 (2013) 018 [arXiv: 1303.2616] [INSPIRE].

[90] M.G. Jackson, B. Wandelt and F. Bouchet, Angular correlation functions for models with logarithmic oscillations, Phys. Rev. D 89 (2014) 023510 [arXiv: 1303.3499] [INSPIRE].

[91] Planck collaboration, P.A.R. Ade et al., Planck 2013 results. XXII. Constraints on inflation, arXiv:1303.5082 [INSPIRE].

[92] P.D. Meerburg, D.N. Spergel and B.D. Wandelt, Searching for oscillations in the primordial power spectrum. I. Perturbative approach, Phys. Rev. D 89 (2014) 063536 [arXiv: 1308.3704] [INSPIRE].

[93] P.D. Meerburg and D.N. Spergel, Searching for oscillations in the primordial power spectrum. II. Constraints from Planck data, Phys. Rev. D 89 (2014) 063537 [arXiv: 1308.3705] [INSPIRE].

[94] M. Aich, D.K. Hazra, L. Sriramkumar and T. Souradeep, Oscillations in the inflaton potential: complete numerical treatment and comparison with the recent and forthcoming CMB datasets, Phys. Rev. D 87 (2013) 083526 [arXiv:1106.2798] [INSPIRE]. 